\title{
The Translational Repressors Nanos and Pumilio Have Divergent Effects on Presynaptic Terminal Growth and Postsynaptic Glutamate Receptor Subunit Composition
}

\author{
Kaushiki P. Menon, ${ }^{1}$ Shane Andrews, ${ }^{2}$ Mala Murthy, ${ }^{1}$ Elizabeth R. Gavis, ${ }^{2}$ and Kai Zinn ${ }^{1}$ \\ 'Division of Biology, California Institute of Technology, Pasadena, California 91125, and ${ }^{2}$ Department of Molecular Biology, Princeton University, \\ Princeton, New Jersey 09544
}

\begin{abstract}
Pumilio (Pum) is a translational repressor that binds selectively to target mRNAs and recruits Nanos (Nos) as a corepressor. In the larval neuromuscular system, Pum represses expression of the translation factor eIF-4E and the glutamate receptor subunit GluRIIA. Here, we show that Nos, like Pum, is expressed at the neuromuscular junction (NMJ) and in neuronal cell bodies. Surprisingly, however, Nos and Pum have divergent functions on both the presynaptic and postsynaptic sides of the NMJ. In nos mutant and nos RNA interference larvae, the number of NMJ boutons is increased, whereas loss of Pum reduces the bouton number. On the postsynaptic side, Nos acts in opposition to Pum in regulating the subunit composition of the glutamate receptor. NMJ active zones are associated with GluRIIA- and GluRIIB-containing receptor clusters. Loss of Nos causes downregulation of GluRIIA and increases the levels of GluRIIB. Consistent with this finding, the electrophysiological properties of NMJs lacking postsynaptic Nos suggest that they use primarily GluRIIB-containing receptors. Nos can regulate GluRIIB in the absence of GluRIIA, suggesting that the effects of Nos on GluRIIB levels are at least partially independent of synaptic competition between GluRIIA and GluRIIB. Nos is a target for Pum repression, and Pum binds selectively to the $3^{\prime}$ untranslated regions of the nos and GluRIIA mRNAs. Our results suggest a model in which regulatory interplay among Pum, Nos, GluRIIA, and GluRIIB could cause a small change in Pum activity to be amplified into a large shift in the balance between GluRIIA and GluRIIB synapses.
\end{abstract}

\section{Introduction}

Nanos (Nos) and Pumilio (Pum) are Drosophila translational repressors that are required for pattern formation during early embryonic development. Pum is a sequence-specific RNAbinding protein that recruits Nos to target mRNAs. Maternally synthesized Pum and Nos repress synthesis of the Hunchback $(\mathrm{Hb})$ transcription factor by binding to sites called Nanos response elements (NREs) in the $3^{\prime}$ untranslated region (UTR) of $h b$ mRNA (for review, see Kuersten and Goodwin, 2003). Pum and Nos also bind to Cyclin B $(C y c B)$ mRNA in the germ cells. Nos represses translation of $C y c B$ mRNA by engaging a deadeny-

Received Jan. 31, 2009; revised Feb. 27, 2009; accepted March 20, 2009.

This work was supported by National Institutes of Health Grants R01 NS28182 (K.Z.) and GM061107 (E.R.G.). We thank Anna Salazar for comments on this manuscript and Ed Silverman (Zinn laboratory) for making Nos protein that was used for antibody generation. We thank Christoph Schuster for pointing out that the minis in our traces for nos mutants had a faster decay rate. We thank Aaron DiAntonio and Stephan Sigrist for helpful advice. We thank Thomas Osterwalder and Haig Keshishian for the muscle GeneSwitch line on the second chromosome, Brett Burke and Haig Keshishian for OK6-GAL4, Vivian Budnik for C57-GAL4, Paul Macdonald for rat Nanos antibody; Peter Bryant for Discs-Large antibody, and Aaron DiAntonio for GluRIIB antibody. We thank Susan Ou of the Caltech Monoclonal Antibody Facility for generating the Nos antibody and the DSHB for synapsin and GluRIIA antibodies. We thank Elena Armand for help with maintaining fly lines. We thank Erin Schuman for the use of her Zeiss LSM510 confocal microscope and Chin-Yin Tai (Schuman laboratory) for advice on quantitation of glutamate receptor puncta. We thank Viola Nesterova for extensive help with figure preparation. Last, we thank anonymous reviewers for comments that greatly improved this manuscript.

Correspondence should be addressed to either Kaushiki P. Menon or Kai Zinn, Division of Biology 114-96, California Institute of Technology, Pasadena, CA 91125. E-mail: menonk@caltech.edu or zinnk@caltech.edu.

D01:10.1523/JNEUROSCI.0520-09.2009

Copyright $\odot 2009$ Society for Neuroscience $\quad$ 0270-6474/09/295558-15\$15.00/0 lase protein complex that removes its polyA tail (Kadyrova et al., 2007). Repression of $h b$ mRNA translation requires another corepressor, Brain Tumor (Brat) (Sonoda and Wharton, 2001), which interacts with the translation initiation machinery (Cho et al., 2006). Pum is also likely to participate directly in repression, because it is a member of the conserved family of Drosophilia pumilio and Caenorhabditis elegans fem-3 mRNA-binding factor (PUF)-domain translational repressors, and PUF proteins can interact with deadenylase/decapping complexes (Wickens et al., 2002; Goldstrohm et al., 2006, 2007; Kadyrova et al., 2007).

Pum and Nos have a variety of functions later in development and appear to work together in most contexts. In the nervous system, Pum represses expression of the Para sodium channel in motor neurons and binds directly to para mRNA. Downregulation of Para by Pum overexpression does not occur in a nos mutant background, suggesting that para mRNA translation is repressed by a Pum/Nos complex (Mee et al., 2004; Muraro et al., 2008). Pum binds to a site in the $3^{\prime}$ UTR of mRNA for the postsynaptic scaffolding protein Discs-large (Dlg), and Pum represses Dlg in mushroom body neurons; Nos has not been assayed in this system (Chen et al., 2008). pum and nos mutants have the same dendritic branching phenotypes in the peripheral nervous system (PNS), and alteration of branching by Nos overexpression requires Pum (Ye et al., 2004). In contrast to these examples, Nos and Pum are proposed to function separately in maintenance of germline stem cells in the ovary (Forbes and 
Lehmann, 1998). The repression targets relevant to stem cell maintenance have not been defined.

In this study, we analyze the functions of Nos at the larval neuromuscular junction (NMJ), which is composed of glutamatergic synapses that use receptors related to the AMPA/kainate family of ionotropic glutamate receptors. Because Nos is necessary for translational repression of all known Pum targets, we had anticipated that loss of Nos would produce phenotypes like those we had previously defined for pum mutants (Menon et al., 2004). Our results, however, show that Nos and Pum act in opposition to each other to regulate glutamate receptor subunit composition and synaptic physiology. Pum represses expression of the GluRIIA subunit of the receptor (Menon et al., 2004), whereas Nos downregulates the alternative subunit GluRIIB. Pum also represses Nos, so that Nos levels are increased when Pum levels go down. Postsynaptic densities that contain mostly GluRIIA receptors allow more current flow in response to transmitter release than do those that are dominated by GluRIIB receptors, and GluRIIA and GluRIIB compete for synaptic occupancy (Petersen et al., 1997; DiAntonio et al., 1999; Sigrist et al., 2002) [but see Schmid et al. (2008) for an alternative view]. This regulatory network, in which Pum represses Nos and GluRIIA, Nos represses GluRIIB, and GluRIIA and GluRIIB antagonize each other, could be used to convert small changes in Pum activity in response to environmental conditions into major alterations in the physiological properties of the NMJ.

\section{Materials and Methods}

\section{Genetics}

"Wild-type" flies used were either $w^{1118}$ or WCS ( $w^{1118}$ crossed to Canton $S)$. nos ${ }^{R C}, n^{R D}$, and deficiency $D f(3 R) D l-F X 1$ were obtained from R. Wharton (Duke University, Durham, NC). Df(3R)Exel6183 was obtained from the Exelixis collection at Harvard University (Boston, MA). GAL4 drivers used in this study were muscle drivers C57-GAL4 (from V. Budnik, University of Massachusetts, Amherst, MA), MHC-GAL4, and 24B-GAL4; the motor neuron driver OK6-GAL4 (from B. Burke and $\mathrm{H}$. Keshishian, Yale University, New Haven, CT); and the pan-neuronal driver C155 (Elav)-GAL4. Controls for RNA interference (RNAi) and overexpression experiments involving drivers were $w^{1118}$ or WCS crossed to the same driver. The Pum RNAi line was obtained from Vienna Drosophila RNAi Center (Dietzl et al., 2007). GluRIIA ${ }^{A D 9}$, GluRIIA and GluRIIB deletions, $S P 22$, and $D f c l-h 4$ were obtained from A. DiAntonio (Washington University, St. Louis, MO).

The muscle driver MHC-GeneSwitch-GAL4213-3 on the second chromosome (from T. Osterwalder and H. Keshishian, Yale University), which has leaky expression in the absence of RU486 (Osterwalder et al., 2001), was used in making the rescue line. The muscle rescue experiments were done by crossing MHC-GeneSwitch GAL4 213-3/CyoActinGFP;nos ${ }^{R D} / T M 3$ Serrate Actin-GFP to UAS-nos-tub3'UTR/CyoActinGFP;Df(3R)Exel6183/TM3 Serrate Actin-GFP, and non-green fluorescent protein (GFP) larvae were selected for analysis. All experiments with nos mutants, muscle rescue lines, and corresponding controls were done with crosses grown at $25^{\circ} \mathrm{C}$. The control and experimental crosses for nos RNAi and overexpression analyses were set up at $29^{\circ} \mathrm{C}$.

To construct a plasmid containing a snapback nos transgene for RNAi analysis, nos sequence from nucleotide 744 to 1203 in exon 2 was inserted into the pWIZ vector on each side of the white intron according to protocols published by Lee and Carthew (2003). We selected two clones: one with inserts oriented head to head and the other in tail-to-tail orientation. Plasmids were sequenced, and transgenic lines were made by standard techniques. The strongest RNAi phenotypes were seen for lines containing the clone that had a tail-to-tail arrangement, as noted previously by Lee and Carthew (2003). We used two transgenic Nos RNAi lines for the experiments in this study: a viable line on the second chromosome and a lethal line on the third chromosome. Nos overexpression was performed by crossing UAS-nos-tub3'UTR ${ }^{31.1}$ (a viable line on the second chromosome) or UAS-nos-tub3'UTR ${ }^{41.1}$ (on the third chromosome) with drivers.

Analysis of GluRIIB levels in larvae where Nos was overexpressed in a GluRIIA mutant background was done by crossing GluRIIA ${ }^{S P 22} /$ Cyo Actin-GFP;MHC-GeneSwitch GAL4/TM3 Serrate Actin-GFP and GluRIIA ${ }^{A D 9} /$ Cyo Actin-GFP;UAS-nos-tub3' UTR ${ }^{41.1} / T M 3$ Serrate Actin-GFP. The control larvae for this experiment were generated by crossing GluRIIA $^{S P 22} /$ Cyo Actin-GFP and GluRIIA ${ }^{A D 9} /$ Cyo Actin-GFP. Early third instar non-GFP larvae were transferred to plates containing $50 \mu \mathrm{g} / \mathrm{ml}$ RU486 for $2 \mathrm{~d}$. They were then dissected and processed for GluRIIB immunohistochemistry.

\section{Antibodies}

Rat anti-Nos was obtained from P. Macdonald (University of Texas, Austin, TX) and used at 1:300. To generate a polyclonal mouse Nos antibody, we made Nos protein in Escherichia coli according to a protocol provided by $\mathrm{R}$. Wharton (Duke University). It was injected into mice at the Caltech Monoclonal Antibody Facility, and the antiserum was used at 1:300. To show that the Nos antibodies recognize Nos protein in situ, we stained larvae that ectopically expressed Nos from GAL4 drivers and demonstrated that the antibodies stained the appropriate patterns.

The other primary antibodies used in this study are listed below. AntiBruchpilot [Brp; nc82 (Development Studies Hybridoma Bank; DSHB)] was used at 1:100; tetramethylrhodamine isothiocyanate-horseradish peroxidase (HRP; Jackson ImmunoResearch Laboratories) was used at 1:50; rabbit anti-Dlg (from P. Bryant, University of California, Irvine, CA) was used at 1:1000; mouse anti-Dlg (DSHB) was used at 1:100; mouse anti-GluRIIA (8B4D2; DSHB) and rabbit anti-GluRIIB (from A. DiAntonio, Washington University) antibodies were used at 1:25 and 1:2000, respectively (Marrus et al., 2004); mouse anti-Synapsin (3C11; DSHB) was used at 1:10; and rabbit anti-Drosophila p21-activated kinase (from N. Harden, University of Toronto, Toronto, Ontario, Canada) was used at 1:2000. Secondary antibodies (Invitrogen) were used at 1:200. Alexa-Fluor 488 anti-mouse and anti-rabbit antibodies were preabsorbed with wild-type embryos and used at 1:500 for Nos antibody staining.

\section{Immunocytochemistry/immunofluorescence}

Third instar larvae were dissected, stained, and processed as described previously (Menon et al., 2004). Briefly, dissected larvae were either fixed in $4 \%$ paraformaldehyde for $30 \mathrm{~min}$ or in Bouin's fix (Sigma) for 1-5 min. Ventral nerve cords (VNCs) were fixed at $4^{\circ} \mathrm{C}$ for $1 \mathrm{~h}$ in $4 \%$ paraformaldehyde. Fixed larval fillets were incubated with primary and secondary antibodies and imaged on a Zeiss LSM510 confocal microscope. All figures shown are maximum intensity projections of confocal $z$-series stacks unless stated otherwise. Images were combined using Adobe Photoshop. Bouton number was quantitated from preparations that were stained with either synapsin or Dlg and anti-HRP antibodies. Maximum intensity projections of confocal $z$-series stacks were analyzed with ImageJ software (National Institutes of Health), and those boutons that showed synapsin labeling with an outline of HRP around the bouton were counted. Muscle sizes were measured from wild-type and mutant larvae, and these showed no significant differences.

For quantitation of GluRIIA and GluRIIB puncta at boutons, larval preparations were either fixed in Bouin's or in cold methanol for $5 \mathrm{~min}$ (for GluRIIA antibody) or in Bouin's for 1 min (for GluRIIB antibody) (Schmid and Sigrist, 2008). Samples were stained either with GluRIIA and anti-HRP antibodies or with GluRIIB and Dlg antibodies. Control and experimental samples were processed together in the same tube and imaged at the same settings on the Zeiss confocal microscope. Z-series stacks were analyzed for intensity measurements using ImageJ software. Confocal stacks were projected by summing all the slices and measuring intensities in both channels at each bouton. For the analysis, three to five terminal boutons from each branch of the muscle $4 \mathrm{NMJ}$ from hemisegments A2 and A3 were quantitated. The background in each channel was determined outside of the NMJ close to the bouton, and that value was subtracted from the mean gray value at the bouton. The GluRIIA and GluRIIB intensity values at each bouton were then normalized with HRP or Dlg intensity measurements. 


\section{Electrophysiology}

For all genotypes, third instar larvae were picked from the walls of the vial and dissected in $\mathrm{Ca}^{2+}$-free HL-3 saline solution. Filleted larvae were washed in solution containing $\mathrm{Ca}^{2+}$ before recording. Recording electrodes were heat-pulled glass capillaries with resistances between 15 and $40 \mathrm{M} \Omega$, filled with $3 \mathrm{M} \mathrm{KCl}$. Miniature excitatory junctional potentials (mEJPs) and evoked EJPs were recorded in the bridge mode using an Axoclamp-2B amplifier and BrownLee preamplifier. The low-pass filter was set at $1 \mathrm{kHz}$ on the preamplifier. Recordings were performed on muscle 6 in abdominal segments 2 and 3. Only those muscles with a resting membrane potential $<60 \mathrm{mV}$ throughout the recording were considered. Spontaneous events were recorded in current clamp for 3 $\mathrm{min}$, and these $\mathrm{mEJP}$ traces were analyzed by hand using the minianalysis program Synaptosoft (by J. Lee; Synaptosoft). mEJP amplitudes and decay times were measured with Synaptosoft. To determine whether the faster decay rate seen in nos mutants was an effect of the events themselves being smaller, we separated mEJP events from both mutant $\left(n o s^{R D} / D f(3 R)\right.$ Exel6183) and wild type $\left(w^{1118}\right)$ into three groups: $\mathrm{mEJP}$ amplitude $<0.5 \mathrm{mV}, \mathrm{mEJP}$ amplitude $0.5-1 \mathrm{mV}$, and $\mathrm{mEJP}$ amplitude 1.5-2 mV. We found that the difference in decay times between wild-type and nos mutant values was similar for all three groups, indicating that the faster decay rate observed in nos mutants was independent of the mEJP amplitude. The decay time distribution was determined using the Synaptosoft program. The first $50 \mathrm{mEJP}$ events from each trace were binned into $5 \mathrm{~ms}$ decay time with bins ranging from $<10$ to $>25 \mathrm{~ms}$. Traces from five animals with a total of 250 events were analyzed for each genotype. For evoked recordings, suction electrodes were fire-polished down to 7 $\mu \mathrm{m}$ tip size and filled with recording solution containing $\mathrm{Ca}^{2+}$. Motoraxons were stimulated with $4 \mu \mathrm{A}$ current at a frequency of $0.2 \mathrm{~Hz}$, and evoked EJPs were recorded from muscle 6 abdominal segment 2 . The average EJP amplitude was calculated from 10 sequential EJPs from each animal. Student's $t$ test was used to determine significance.

\section{RNA binding assays}

Plasmid construction. Plasmids used for synthesis of GluRIIA (wild-type), $h b$, and nos RNA probes were generated by inserting annealed oligos (with blunt ends) into the SmaI site of the pBluescript derivative pBSSK $\Delta K P$ (Kalifa et al., 2006). Sense strand sequences are as follows: GluRIIA, 5'GATCGGTGTCCACATTGTATATTGTGAGCTATATTTGTAGAATTATTTT3'; $h b$, NRE 5'GATCTATTATTTTGTTGTCGAAAATTGTACATAAGCCT; $h b \mathrm{NRE}^{-}, 5^{\prime}$ GATCACTATCATAAAGACTAGTCTGGAGAAACATT3'; nos (wild type), 5'GATCCACTTTGAATTTCGAACTGTCAATCGTATCATTAGAATTTAATCTT3'. The GluRIIA GUUA and nos M1, M2, and M3 mutant plasmids are based on GluRIIA and nos wild type but contain the sequence changes shown in Figure $10 \mathrm{~A}$. For the GluRIIA M2 plasmid, annealed oligos (5'GATCCCGGTGTCCACATTGTATCTGAGACGCTATATTTGTAGAATTATTTTA3' and 5'CTAGTAAAATAATTCTACAAATATAGCGTCTCAGATACAATGTGGACACCGG3') were cloned between the BamHI and SpeI sites of pBSSK $\Delta K$ K. Plasmids used to synthesize nos 3'UTR probes for UV-crosslinking have been described previously (Gavis et al., 1996a; Bergsten et al., 2001).

Purification of Pum RNA-binding domain. His-tagged Pum RNAbinding domain (PumRBD) was expressed in E. coli BL21(DE3)pLysE at $29^{\circ} \mathrm{C}$. The cell pellet was resuspended in lysis buffer ( $50 \mathrm{~mm}$ Tris- $\mathrm{HCl}, \mathrm{pH}$ 8.0, $100 \mathrm{~mm} \mathrm{NaCl}, 5 \mathrm{~mm}$ EDTA) containing 0.5\% Triton X-100, $0.1 \mathrm{~mm}$ PMSF, and $1 \mathrm{~mm}$ DTT and sonicated four times for $1 \mathrm{~min}$. The lysate was supplemented to $10 \mathrm{~mm} \mathrm{MgSO}_{4}$, and DNase (to $10 \mu \mathrm{g} / \mathrm{ml}$ ) and lysozyme (to $0.1 \mathrm{mg} / \mathrm{ml}$ ) were added, followed by incubated at room temperature for $20 \mathrm{~min}$. The lysate was cleared by centrifugation for $10 \mathrm{~min}$ at 5000 $\mathrm{rpm}$. The supernatant was passed twice over a Ni-NTA agarose (Qiagen) column equilibrated with lysis buffer. After washing with $40 \mathrm{~mm}$ imidazole/50 mM Tris-HCl, pH 8.0, bound protein was eluted with $400 \mathrm{~mm}$ imidazole $/ 50 \mathrm{~mm}$ Tris- $\mathrm{HCl}, \mathrm{pH}$ 8.0. Pooled eluate fractions were passed over a PD10 column equilibrated to $50 \mathrm{~mm}$ Tris- $\mathrm{HCl}, \mathrm{pH}$ 7.5/200 mM $\mathrm{KCl} / 1 \mathrm{~mm}$ DTT/1 mм EDTA/10\% glycerol. Purified protein was concentrated to $1 \mathrm{mg} / \mathrm{ml}$ by centrifugal filtration (Centricon), aliquoted, and stored at $-80^{\circ} \mathrm{C}$

In vitro binding assays. Linearized template DNA was transcribed with ${ }^{32} \mathrm{P}$-dUTP as described previously (Bergsten et al., 2001). Before use,
${ }^{32} \mathrm{P}$-labeled probes were denatured by heating at $65^{\circ} \mathrm{C}$ for $5 \mathrm{~min}$ and placed on ice. Gel mobility shift assays were performed as described previously (Zamore et al., 1997), with the omission of Tween 20 from the buffer. Competition assays were performed in an identical manner with the addition of cold competitor RNA as indicated in the figure legend. UV-crosslinking assays were performed as described previously (Bergsten et al., 2001) using the same binding buffer as for gel mobility shift assays.

\section{Results}

\section{Nos is localized to larval NMJs}

To determine whether the distribution of zygotically expressed Nos protein is similar to that of Pum, we localized Nos by immunofluorescence using two antibodies generated by different groups in different species. Both were shown to recognize ectopically expressed Nos protein in situ (see Materials and Methods for details). We focused on sites where Pum is expressed in third instar larvae, including the NMJ, muscles, and VNC. All images shown here are of NMJs in abdominal segments A2 or A3, visualized by confocal microscopy.

At the NMJ, we found that Nos colocalized with the neuronal marker recognized by anti-HRP antibody. Type $1 \mathrm{~b}$ (big; arrow) and type 1s (small; arrowhead) NMJ boutons are both glutamatergic and are from different neurons (Fig. 1A1). Nos was observed at both types of boutons, using either antibody for staining (Fig. $1 A, B$ ). Examination of a single confocal slice of an NMJ (Fig. $1 G$ ) shows that Nos labeling is primarily confined within the bouton boundaries outlined by the neuronal marker. As described below, our RNAi results show that most of the Nos at the $\mathrm{NMJ}$ is postsynaptic, because it derives from expression in muscles. This suggests that Nos is localized to the postsynaptic subsynaptic reticulum (SSR) immediately under and around the bouton, so that a border between anti-HRP and Nos cannot be distinguished by confocal microscopy. Nos was also observed in neuronal cell bodies in the VNC (Fig. 1D). The outlines of antiHRP-labeled cell bodies coincide with Nos staining, indicating that Nos is present in neuronal soma. Nos is absent from axons, which are brightly labeled by anti-HRP.

For comparison, we have included some of our earlier data showing Pum localization at the NMJ (Fig. 1C). Pum staining has a more "postsynaptic" appearance than Nos staining at the NMJ, because Pum colocalizes with the SSR marker Dlg. We also showed that Pum staining forms rings around the presynaptic marker Synaptotagmin (Menon et al., 2004).

Because proteins reactive with anti-Nos antibodies continue to be expressed in strong nos mutants (Verrotti and Wharton, 2000), we could not use reduced staining of mutants to demonstrate that Nos localization to the NMJ is authentic. Therefore, we analyzed larvae in which Nos expression was knocked down using RNAi. We generated transgenic flies expressing a hairpin nos RNAi under control of the yeast upstream activating sequence (UAS), which binds to the GAL4 transcription factor. Nos double-stranded RNA is expressed only when a GAL4 source is provided by crossing a Nos RNAi line to a GAL4 "driver" line (Brand and Perrimon, 1993). To examine protein expression, we crossed a strong muscle driver, C57-GAL4, to Nos RNAi lines and to wild-type control flies and analyzed larvae from these crosses by staining with Nos antibody. Nos staining at the NMJ was markedly decreased in muscle Nos RNAi larvae (Fig. 1, compare E1, F1), whereas anti-HRP staining (Fig. 1 E2,F2) was unchanged. Similar results were obtained for other NMJs (data not shown). These results show that most of the Nos protein that is localized to the NMJ derives from expression in postsynaptic muscle fibers.

To analyze neuronal Nos expression, we crossed a Nos RNAi 

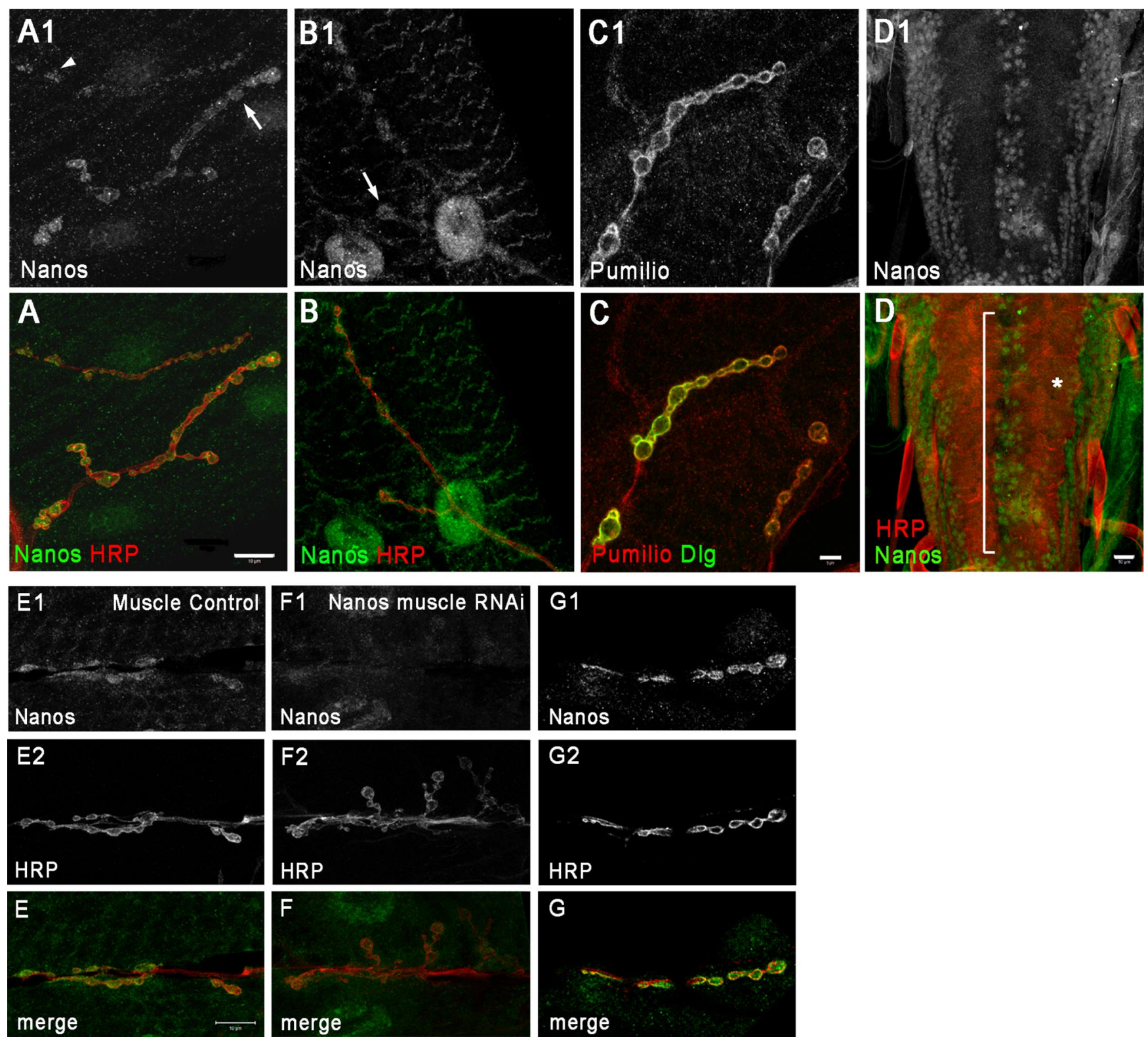

Figure 1. Nos is localized to the larval NMJ. $\boldsymbol{A}-\boldsymbol{C}$, Muscle $4 \mathrm{NMJ}$ of $A 2$ segments stained with mouse Nos antibody ( $\boldsymbol{A}$, green in $\boldsymbol{A})$, rat Nos antibody (B1, green in $\boldsymbol{B})$, and Pum antibody ( $\boldsymbol{C}$, red in $\boldsymbol{C}$. Double labeling with anti-HRP in red $(\boldsymbol{A}, \boldsymbol{B})$ and with anti-Dlg in green $(\boldsymbol{C})$ is shown. Type $1 \mathrm{~b}$ boutons are indicated by arrows $(\boldsymbol{A} \mathbf{1}, \boldsymbol{B} \mathbf{1})$, and type 1 s boutons are indicated by the arrowhead $(\boldsymbol{A} \mathbf{1})$. $\boldsymbol{D}$, Larval VNC staining with mouse Nos antibody ( $\mathbf{D}$ 1, green in $\boldsymbol{D}$ ) and anti-HRP (red in $\boldsymbol{D}$ ). The asterisk indicates axons, and the bracket shows medial neurons that stain with Nos antibody and anti-HRP. $\boldsymbol{E}, \boldsymbol{F}$, Muscle 6/7 NMJs of A3 segments stained with mouse Nos antibody $(\boldsymbol{E} 1, \boldsymbol{F} 1)$ and anti-HRP $(\boldsymbol{E 2}, \boldsymbol{F 2})$. $\boldsymbol{E}$ and $\boldsymbol{F}$ are merged images. The muscle control NMJ (E) is from C57-GAL4 crossed to $w^{1118}$. The muscle Nos RNAi NMJ $(\boldsymbol{F})$ is from C57-GAL4 crossed to Nos RNAi. Note the reduction in staining in $\boldsymbol{F} \mathbf{1}$ compared with $\boldsymbol{E 1}$; the anti-HRP signal is the same in $\boldsymbol{E 2}$ and $\boldsymbol{F 2}$. All panels are confocal $z$-series projections, except for $\mathbf{D 1}, \mathbf{D}, \mathbf{G 1}$, and $\mathbf{G}$, which are single sections. $\mathbf{G}$, A cross section of a 6/7 A2 NMJ labeled with mouse Nos antibody $(\mathbf{G 1}$, green in $\boldsymbol{G})$ and anti-HRP (G2, red in $\mathbf{G}$ ). Scale bars: $A, D, E, 10 \mu \mathrm{m} ; C, 5 \mu \mathrm{m}$.

line to the pan-neuronal driver C155 (Elav)-GAL4 (supplemental Fig. S1, available at www.jneurosci.org as supplemental material). Well defined outlines of neuronal cell bodies labeled with Nos antibody in the control larval CNS (Fig. 1D) were absent in animals in which neuronal Nos was knocked down (supplemental Fig. S1, available at www.jneurosci.org as supplemental material). Although staining of cell bodies was reduced, staining of NMJs in neuronal RNAi larvae was unaffected (supplemental Fig. S2, available at www.jneurosci.org as supplemental material).

\section{Neuronal Nos regulates bouton number and NMJ branching} For analyzing nos mutant phenotypes, we used transheterozygotes of the strong nos alleles $n o s^{R C}$ and $n o s^{R D}$ in combination with two deficiency (Df) mutations, $D f(3 R)$ Exel6183 and $D f(3 R) D l-F X 1$, which delete the nos gene. nos ${ }^{R C}$ is a point mutation in the splice donor of intron 1, and nos ${ }^{R D}$ (Cys354Tyr) is a missense mutation in the conserved C-terminal domain (Curtis et al., 1997). nos ${ }^{R D}$ encodes an unstable protein, whereas nos ${ }^{R C}$ mutant expresses low levels of full-length protein (Verrotti and Wharton, 2000). Both nos ${ }^{R C}$ and $n o s^{R D}$ produce strong maternal phenotypes affecting abdominal segmentation and germ cell migration. Zygotic nos ${ }^{R C}$ mutants have defects in dendritic branching in the PNS (Ye et al., 2004). In our hands, neither allelic combination resulted in a reduction in Nos staining in the larval CNS or at the NMJ. In fact, Nos protein at the NMJ was increased in these transheterozygotes (see below). 

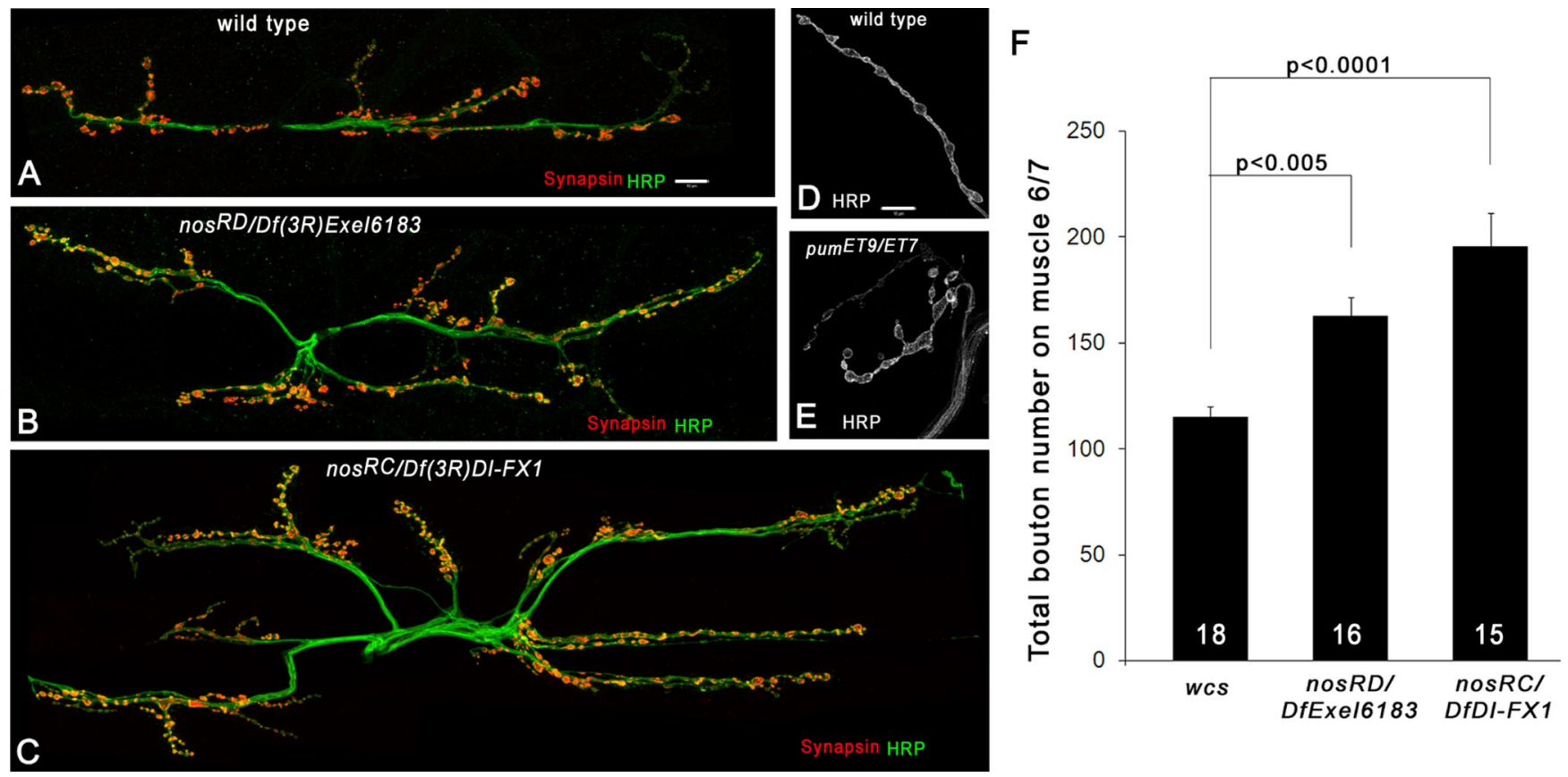

Figure 2. Nos mutant NMJs have an increased number of synaptic boutons. A-C, Muscle 6/7 NMJs of A2 segments labeled with synapsin (red) and anti-HRP (green) antibodies in wild-type (WCS) and two nos mutant transheterozygotes, nos ${ }^{R D} / D f(3 R)$ Exel6183 and nos ${ }^{R C} / D f(3 R) D I-F X 1 . D, E$, Muscle 4 NMJs of A3 segments labeled with anti-HRP antibody in wild type $\left(w^{1118}\right)$ and $p u m^{E T 9} /$ pum $^{E T 7}$. Note the large boutons and the fused bouton phenotype in $\boldsymbol{E}$. F, A graph of the total bouton number at 6/7 A2 NMJs for three genotypes: WCS, nos ${ }^{R D} / D f(3 R) E x e l 6183$, and nos ${ }^{R C} / D f(3 R) D I-F X 1$. The differences between WCS and nos mutants are highly significant ( $p<0.005$ and $p<0.0001$, Student's $t$ test). Scale bars: $A, D, 10 \mu \mathrm{m}$.

To study how loss of Nos function affects NMJ structure, we examined third instar NMJ architecture and bouton morphology in nos mutants. It is apparent that there are more boutons at NMJs in mutants compared with those in control larvae (Fig. $2 A-C)$. Muscle sizes in mutants were not significantly different from controls. Quantitation of bouton number in the three genotypes showed that the strongest effect is observed in $n o s^{R C} / D f$ (3R)Dl-FX1, with a 1.7-fold increase in bouton number relative to control larvae (wild-type wcs: $114.9 \pm 4.9$ boutons/NMJ, $n=$ 18; $\operatorname{nos}^{R D} / D f(3 R)$ Exel6183: $162.6 \pm 9, n=16$; $\operatorname{nos}^{R C} / D f(3 R) D l$ FX1: $195.3 \pm 15.9, n=15$ ) (Fig. $2 F$ ). These phenotypes are different from those we previously defined for pum mutants, which are described in detail in published work (Menon et al., 2004). Boutons in pum mutants are irregularly shaped, fewer in number, and larger in size than those at control NMJs (Fig. $2 D, E)$. In contrast, boutons on all muscles in nos mutants have relatively normal sizes and shapes.

We then asked whether the phenotypes seen in nos mutants were caused by the loss of Nos function in the neurons, in the muscles, or both. To address this, we crossed our Nos RNAi lines to the pan-neuronal driver C155-GAL4, resulting in a reduction in Nos protein only in neurons. Figure 3 shows muscle 4 NMJs of control larvae [C155-GAL4 crossed to $\left.w^{1118}(\mathrm{~A})\right]$ and neuronal Nos RNAi larvae [C155-GAL4 crossed to Nos RNAi (B)] (two independent RNAi insertions were tested, on the second and third chromosomes, and they produced similar phenotypes). Quantitation of bouton numbers on muscle 6/7 of F1 larvae showed that RNAi knockdown of Nos in neurons resulted in a 1.6-fold increase in the number of boutons compared with neuronal control NMJs (C155-GAL4 crossed to wild type; control: 124.1 \pm 4.8, $n=11$; neuronal Nos RNAi: $202.3 \pm 5.7, n=12$; Student's $t$ test, $p<0.0001$ ) (Fig. $3 G$ ). Bouton numbers were also increased by 1.3 -fold, however, at NMJs of muscle Nos RNAi larvae (24B-GAL4 crossed to Nos RNAi) compared with control animals (control: $137.3 \pm 7.7, n=15$; muscle Nos RNAi: $183.8 \pm$ 9.8, $n=12$; Student's $t$ test, $p<0.001$ ) (Fig. $3 D, E, G$ ). 24B-GAL4 is another early muscle-specific GAL4 driver, similar in strength to C57-GAL4. These data suggest that the increase in bouton number observed in nos mutants is a consequence of reduction in both presynaptic and postsynaptic Nos functions.

We also examined the effects of overexpressing Nos in neurons or in muscles on bouton number. This was done by crossing a motor neuron-specific driver, OK6-GAL4, or a muscle driver, 24B-GAL4, to a transgenic line bearing a construct in which the nos 3'UTR was replaced with the tubulin (tub) 3'UTR (nostub3'UTR) (Gavis et al., 1996b). This transgene lacks all nos $3^{\prime} \mathrm{UTR}$ regulatory sequences and therefore expresses Nos at high levels when crossed to drivers.

Neuronal overexpression of Nos produced boutons with irregular rounded shapes and caused a slight increase in bouton numbers (neuronal control: $184 \pm 9.3, n=13$; neuronal Nos overexpression: $222 \pm 12.4, n=14$ ) (Fig. $3 C$ ). In contrast, neuronal overexpression of Pum generates abnormal NMJs composed of numerous tiny boutons compacted into a small area (Menon et al., 2004). Muscle overexpression of Nos produced dramatic changes in the morphology of NMJs (Fig. 3F). It was not possible to count boutons in these NMJs because of their abnormal clustering.

Perturbation of Nos expression in neurons affects active zone numbers in type 1s boutons

A closer examination of one of the two types of glutamatergic boutons, type 1s, in neuronal Nos overexpression and RNAi larvae showed interesting alterations in the distribution of active zones (AZs). AZs were visualized with the Brp marker (Kittel et al., 2006; Wagh et al., 2006) (Fig. 3H). The numbers of Brp puncta in type 1s boutons (recognized by weak staining for the SSR marker Dlg) are reduced in neuronal Nos overexpression 


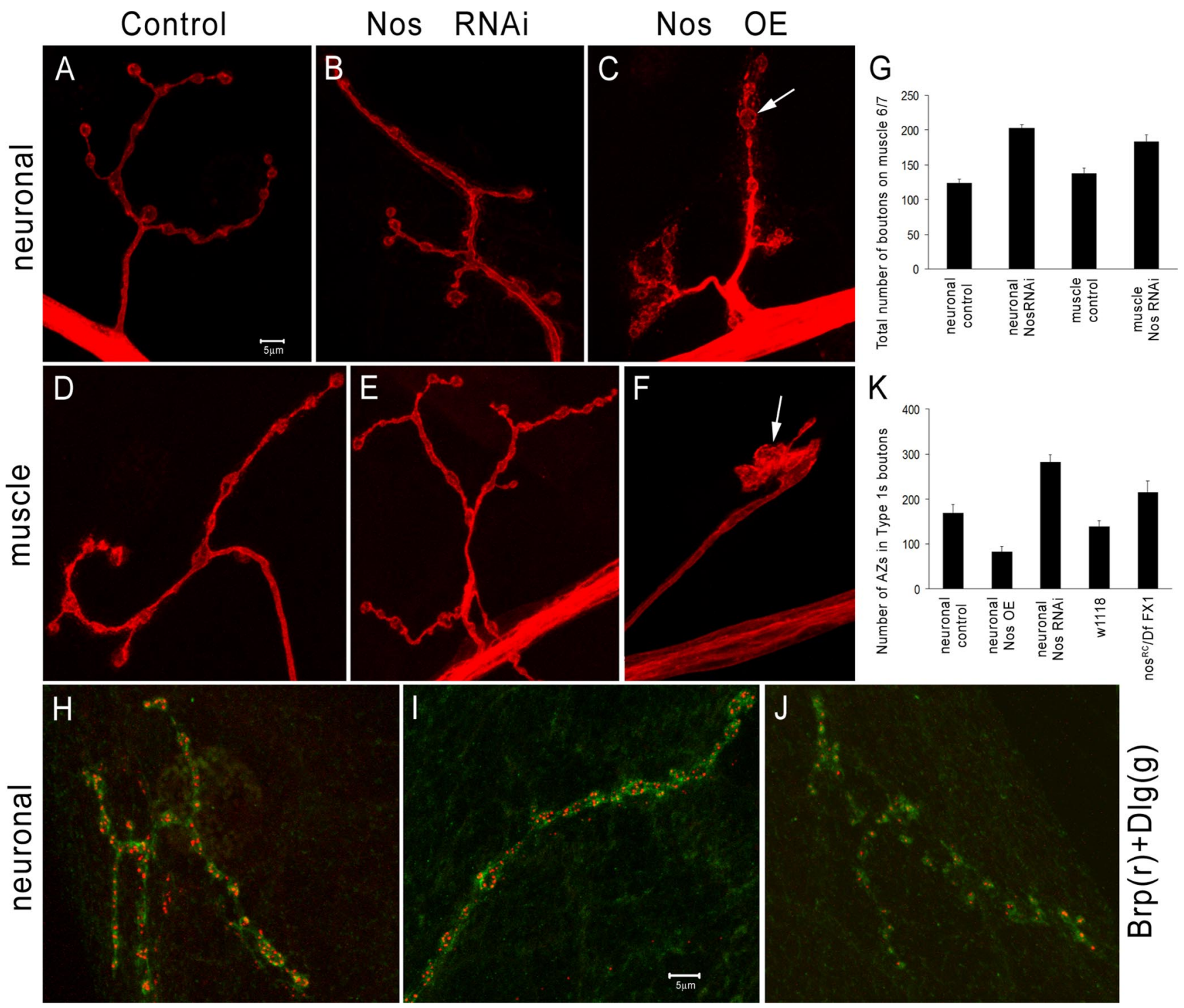

Figure 3. Perturbation of Nos alters bouton and AZ numbers. $\boldsymbol{A}-\boldsymbol{F}$, Muscle 4 NMJs of A2 segments labeled with anti-HRP (red) antibody for neuronal ( $\boldsymbol{A}-\boldsymbol{C}$ ) and muscle (D-F) Nos RNAi and Nos overexpression. $\boldsymbol{A}-\boldsymbol{C}$, Neuronal control (C155-GAL4 crossed to $\left.\boldsymbol{W}^{1118} ; \boldsymbol{A}\right)$, C155-GAL4 crossed to Nos RNAi (B), and OK6-GAL4 crossed to a UAS-Nanos line (C, Nos 0E). Neuronal drivers $(155-$-GAL4 and OK6-GAL4 produced similar phenotypes when used to overexpress Nos. The arrow in $\mathbf{C}$ shows an irregularly shaped type $1 \mathrm{~b}$ bouton. $\boldsymbol{D}-\boldsymbol{F}$, Muscle control (24B-GAL4 crossed to $\left.\mathbf{W}^{1118} ; \boldsymbol{D}\right)$, muscle knockdown of Nos (24B-GAL4 crossed to a Nos RNAi line; $\boldsymbol{E}$ ), and muscle overexpression of Nos (Nos OE; 24B-GAL4 crossed to a UAS-Nanos line; $\boldsymbol{F}$ ). Note the abnormal structure of the entire muscle 4 NMJ when Nos is overexpressed in muscles ( $\boldsymbol{F}$, arrow). $\boldsymbol{G}$, A graph of the total bouton number at muscle $6 / 7$ clefts in neuronal control ( $n=11)$, neuronal Nos RNAi ( $n=12)$, muscle control ( $n=$ $15)$, and muscle Nos RNAi $(n=12)$ larvae. The difference in bouton number between NMJs of neuronal control and neuronal Nos RNAi larvae and between muscle control and muscle Nos RNAi are highly significant (Student's $t$ test; $p<0.0001$ and $p<0.001$, respectively). $\boldsymbol{H}-\mathbf{J}$, Type 1s boutons of muscle 4 NMJs in A2 segments labeled for the AZ marker Brp (red) and Dlg (green) are shown for neuronal control $(\boldsymbol{H})$, neuronal Nos RNAi $(\boldsymbol{I})$, and neuronal Nos overexpression $(\boldsymbol{J})$. $\boldsymbol{K}$, The total number of AZs in type 1s boutons of muscle 4 NMJs in A2 segments were quantitated in five genotypes: neuronal control $(n=9)$, neuronal Nos overexpression (Nos $0 \mathrm{E} ; n=7)$, neuronal Nos RNAi $(n=8)$, wild type $\left(w^{1118} ; n=5\right)$, and nos ${ }^{R C} / D f(3 R) D I-F X 1(n=7)$. The differences between control, neuronal OE (Student's $t$ test, $p<0.005$ ), and neuronal RNAi $(p<0.0005)$ lines are highly significant. Scale bars: $A, I, 5 \mu \mathrm{m}$.

larvae (Fig. 3J). The total number of AZs (Brp puncta) in the entire type 1 s arbor of muscle 4 NMJs was decreased by twofold compared with controls (control, $168.2 \pm 19.3$; neuronal Nos overexpression larvae, $82.6 \pm 11.6$ ) (Fig. $3 J, K$ ). We also scored the number of Brp-marked AZs per type 1s bouton and found that neuronal Nos overexpression larvae had a threefold increase in the fraction of type 1s boutons that had only one AZ (11.7 \pm 2.4 boutons with one AZ in control muscle 4 NMJs; $35.7 \pm 2.2$ boutons with one $\mathrm{AZ}$ in neuronal Nos overexpression). The number of Brp puncta in type $1 \mathrm{~b}$ boutons of neuronal Nos overexpression larvae were not significantly different from controls (data not shown).

Having noted the decrease in Brp-marked AZs conferred by neuronal overexpression of Nos, we wondered whether reduction of Nos in neurons would also produce an AZ phenotype. We found that knockdown of neuronal Nos with RNAi resulted in the opposite effect, producing a 1.7-fold increase in AZs (Brp puncta) in type 1s boutons relative to controls (neuronal Nos RNAi, $282.5 \pm 15.7$ ) (Fig. $3 I, K)$. An increase was also seen in nos mutants (wild type, 138.6 $\pm 13.1 ;$ nos $^{R C} / D f(3 R) D l-F X 1,215.1 \pm$ 25.9) (Fig. 3K).

Postsynaptic Nos regulates GluRIIA and GluRIIB, acting in opposition to Pum

We next analyzed the functions of Nos on the postsynaptic side of the NMJ. GluRIIA is dramatically upregulated in pum mutants 


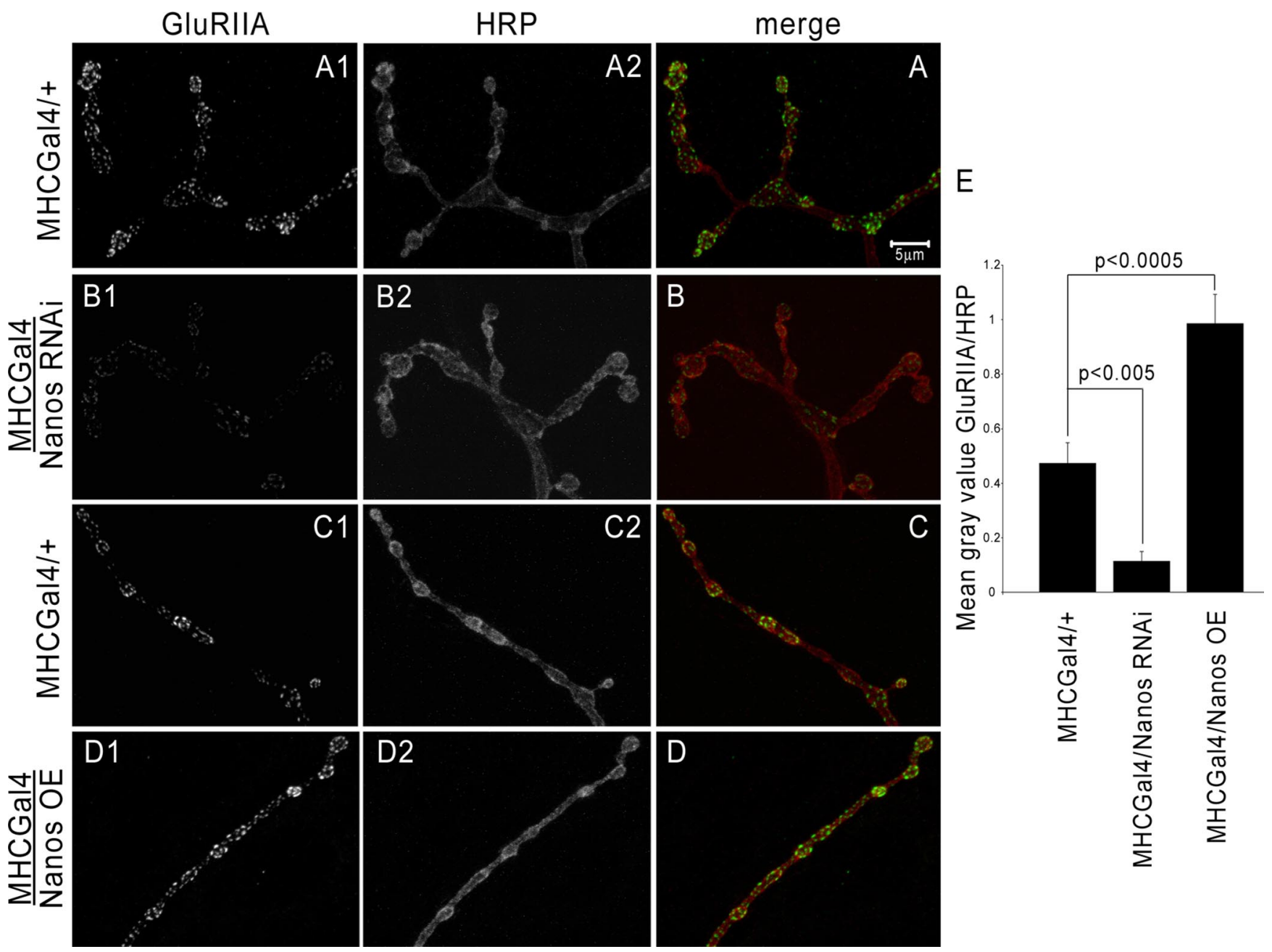

Figure 4. Knockdown of postsynaptic Nos causes downregulation of GluRIIA, and Nos overexpression upregulates GluRIIIA. $A-D$, Muscle 4 NMJs in $A 2$ segments labeled with anti-GluRIIIA and anti-HRP. A, C, Control NMJs (MHC-GAL4 crossed to $\boldsymbol{W}^{1118}$ ). B, An NMJ from Nos RNAi crossed to MHC-GAL4. D, An NMJ from UAS-Nos crossed to MHC-GAL4 (Nanos 0E). $A, B, G$, GluRIIIA levels are decreased when Nos is knocked down in muscles (compare $\boldsymbol{A} \mathbf{1}, \boldsymbol{B} \mathbf{1}$; green in $\boldsymbol{A}$ and $\boldsymbol{B}$ ). Anti-HRP (neuronal marker) levels are similar in both control and Nos RNAi NMJs ( $\boldsymbol{A 2}, \boldsymbol{B 2}$; red in $\boldsymbol{A}$ and $\boldsymbol{B}$ ). $\boldsymbol{C}$, $\mathbf{D}$, GluRIIA levels are increased when Nos is overexpressed in muscles (compare $\mathbf{C}, \mathbf{D}$; green in $\mathbf{C}$ and $\mathbf{D})$. Anti-HRP labeling is similar in control and overexpression larvae $(\mathbf{C}, \mathbf{D 2}$; red in $\mathbf{C}$ and $\mathbf{D}$ ). Two control NMJs are shown in this figure because the larvae for the RNAi or the overexpression crosses were processed and imaged at the same time along with their corresponding control animals in the same tube. Scale bar: $\boldsymbol{A}, 5 \mu \mathrm{m}$. $\boldsymbol{E}$, Quantitation of GluRIIA at muscle 4 NMJs in control, Nos RNAi, and Nos overexpression (Nos OE). GluRIIA levels in distal boutons were quantitated and normalized against anti-HRP. This is represented as mean gray value of GluRIIA/HRP per hemisegment (see Materials and Methods). The values for MHC-GAL4/Nos RNAi (0.11 $\pm 0.03, n=16)$ and MHC-GAL4/Nos $0 \mathrm{E}(0.98 \pm 0.11, n=16)$ were significantly different from control MHC-GAL4/ $+(0.47 \pm 0.08, n=35)$ (Student's $t$ test; $p<0.005$ and $p<0.0005$, respectively).

(Menon et al., 2004) (see Fig. 8). If Nos acts as a Pum corepressor in regulating GluRIIA translation, we would expect to see a similar increase in GluRIIA when postsynaptic Nos is knocked down. In these experiments, we examined larvae in which Nos was depleted from muscles using RNAi or was overexpressed in muscles using the nos-tub3'UTR transgene. For these muscle RNAi and overexpression experiments, we used the myosin heavy chain (MHC)-GAL4 driver, which is switched on in somatic muscles in first instar larvae and remains active throughout larval life. This was because Nos overexpression from strong, early muscle drivers such as 24B- and C57-GAL4 alters NMJ morphology (Fig. $3 F$ ), whereas MHC-GAL4-driven expression does not. We crossed MHC-GAL4 with control or Nos RNAi lines and analyzed F1 larvae from these crosses by anti-GluRIIA immunofluorescence. Surprisingly, we saw a decrease in GluRIIA immunoreactivity relative to control larvae [Fig. 4, compare A1, B1 (green in $A$ and $B)$ ] when Nos was knocked down in the muscles with RNAi. Conversely, Nos overexpression resulted in an increase in GluRIIA levels [Fig. 4, compare C1, D1 (green in $C$ and D)].
Perturbation of Nos expression had no effect on expression of the anti-HRP marker used for double staining of the NMJs.

To quantitatively assess these effects, we measured the average fluorescence intensity of GluRIIA at distal boutons, normalizing these values to the intensity of the marker used for double staining (see Materials and Methods for details). This measurement reflects both the number of GluRIIA puncta and their individual intensities. Larvae used for quantitation were processed and imaged together under the same experimental conditions. We found that RNAi knockdown decreases GluRIIA by fourfold, whereas overexpression increases it by twofold. The values for MHC-GAL4/Nos RNAi $(0.11 \pm 0.03 ; n=16)$ and MHC-GAL4/ Nos OE $(0.98 \pm 0.11 ; n=16)$ were significantly different from control MHC-GAL4/ $+(0.47 \pm 0.08 ; n=35)$ (Student's $t$ test, $p<0.0005$ and $p<0.005$, respectively).

The ionotropic muscle glutamate receptor in Drosophila consists of five known subunits: GluRIIA, GluRIIB, GluRIIC, GluRIID, and GluRIIE (Marrus et al., 2004; Qin et al., 2005). A functional receptor is composed of either IIA or IIB in addition to 

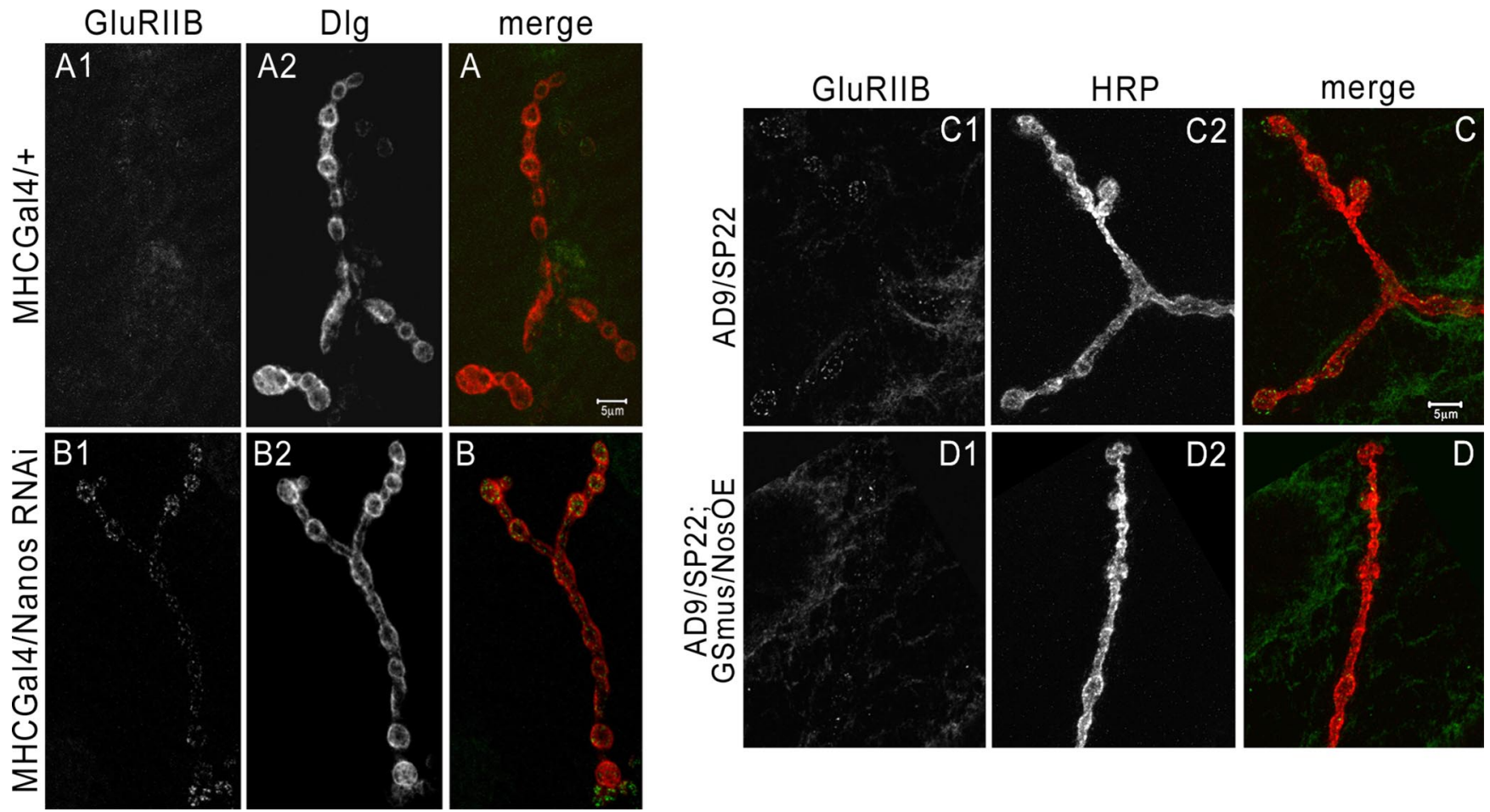

Figure 5. Knockdown of Nos in muscles upregulates GluRIIB, and Nos overexpression in muscles in a GluRIIA mutant downregulates GluRIIB. $A, B$, Muscle 4 NMJs in A2 segments labeled with anti-GluRIIB and anti-Dlg. MHC-GAL4/+ $(\boldsymbol{A})$ is a control NMJ (MHC-GAL4 muscle driver crossed to $\boldsymbol{w}^{1118}$ ), and MHC-GAL4/Nos RNAi $(\boldsymbol{B})$ is a Nos RNAi line crossed to MHC-GAL4. GluRIIB levels are increased when Nos is knocked down in muscles (compare $\boldsymbol{A} \mathbf{1}, \mathbf{B} \mathbf{1}$; green in $\boldsymbol{A}$ and $\boldsymbol{B}$ ). Dlg labeling is the same in control and Nos RNAi NMJs ( $\boldsymbol{A 2}, \boldsymbol{B} 2$; red in $\boldsymbol{A}$ and $\boldsymbol{B})$. GluRIIB labeling from distal boutons at muscle 4 NMJs was quantitated (see Materials and Methods), and these values were normalized against Dlg intensity. The mean gray value of GluRIlB/Dlg per hemisegment for MHC-GAL4/Nos RNAi was $0.41 \pm 0.1(n=18)$, significantly different from control MHC-GAL4/ $+(0.14 \pm 0.08 ; n=18$; Student's $t$ test, $p<0.02)$. C, $\boldsymbol{D}$, Muscle 4 NMJs in A2 segments labeled with anti-GluRIIB and anti-HRP. C, Control NMJ, AD9/SP22, is a transheterozygote of a GluRIIA null mutant GluRIIAAD over a deficiency GluRIIA ${ }^{\text {SP22 }}$ that removes both GluRIIA and GluRIIB. D, AD9/SP22; GSmus/NosOE is an AD9/SP22 animal in which Nos is overexpressed from the MHC-GeneSwitch-GAL4 driver. Note that GluRIIB levels are decreased at the boutons of the NMJ in animals where Nos is overexpressed in a GluRIIA mutant (compare D1, C1; green in $\mathbf{C}$ and $\mathbf{D}$ ). The magnitude of the decrease is approximately threefold (see Results). HRP labeling is the same in both control and experimental $\operatorname{NMJs}(\mathbf{C}, \mathbf{D} 2$; red in $\mathbf{C}$ and $\boldsymbol{D})$. Scale bars: $\boldsymbol{A}, \boldsymbol{C}, 5 \mu \mathrm{m}$.

subunits IIC, IID and IIE, which are invariant. Channel properties differ between receptors with a IIA subunit and those with a IIB subunit (Petersen et al., 1997; DiAntonio et al., 1999) (see below for further discussion). The decrease in GluRIIA caused by Nos knockdown motivated us to examine GluRIIB levels as well.

When Nos was knocked down in muscles, the amounts of GluRIIB at NMJ boutons [Fig. 5, compare $A 1, B 1$ (green in $A$ and $B)]$ were increased compared with control larvae. Quantitation of GluRIIB at muscle 4 NMJs in control and Nos RNAi showed that RNAi knockdown produced a threefold increase in GluRIIB. GluRIIB labeling from distal boutons was quantitated and normalized against Dlg. This is represented as the mean gray value of GluRIIB/Dlg per hemisegment (see Materials and Methods). The values for MHC-GAL4/Nos RNAi $(0.41 \pm 0.1 ; n=18)$ were significantly different from control MHC-GAL4/+ $(0.14 \pm 0.08$; $n=18$ ) (Student's $t$ test, $p<0.02$ ).

GluRIIA and GluRIIB receptors fluctuate in opposition to each other, such that a decrease in synaptic GluRIIA receptors at the NMJ is coupled to an increase in GluRIIB receptors, and vice versa (Petersen et al., 1997; DiAntonio et al., 1999; Sigrist et al., 2002). This suggests that the observed increase in GluRIIB in Nos RNAi knockdown larvae could be an indirect consequence of the reduction in GluRIIA that occurs in these larvae. To address this question, we examined GluRIIB in larvae that are heterozygous for a GluRIIA null mutation over a deficiency that removes both GluRIIA and GluRIIB (these genes are closely linked). Because GluRIIB should already be upregulated in these larvae, we did not examine the effect of Nos RNAi on its levels, but rather asked whether Nos overexpression could reduce GluRIIB. This is a difficult experiment because of the low signal-to-noise ratio often observed with the anti-GluRIIB antibody. However, we were able to show that Nos overexpression reduced GluRIIB levels by threefold (Student's $t$ test, $p<0.005$ ) (Fig. 5, compare C1, D1 (green in $C$ and $D$ )]. These data indicate that the regulation of GluRIIB by Nos is at least partially independent of GluRIIA.

Electrophysiological nanos phenotypes are consistent with an increase in GluRIIB-dominated synapses

To evaluate the functional relevance of the control of GluRIIB by Nos, we recorded from the NMJs of nos mutants (Fig. 6). nos mutants had a $40 \%$ decrease in mEJP (mini) amplitude compared with wild-type controls (control: $1.01 \pm 0.01 \mathrm{mV}, n=1$; $n o s^{R D} / D f(3 R)$ Exel 6183, $0.61 \pm 0.003 \mathrm{mV}, n=26$; Student's $t$ test, $p<0.0001$ ) (Fig. 6A). The decrease in mini amplitude was partially rescued by muscle expression of Nos in the mutant background (muscle rescue: $0.82 \pm 0.06 \mathrm{mV}, n=14$ ). The difference between mutant and muscle rescue values is highly significant (Student's $t$ test, $p<0.0001$ ). We also confirmed that Nos in the muscle is primarily responsible for the decrease in mini amplitude by knocking down Nos in larval muscles with RNAi. The nos mutant phenotype was recapitulated in muscle Nos RNAi larvae, as mini amplitudes were decreased by $40 \%$ compared with control larvae (control: $0.82 \pm 0.06 \mathrm{mV}, n=15$; muscle Nos RNAi: $0.50 \pm 0.003 \mathrm{mV}, n=15$; Student's $t$ test, $p<0.0001$ ) (Fig. 6A). Representative mEJP traces for all genotypes are shown in Fig. $6 E$. We also examined mini amplitudes in neuronal Nos RNAi 

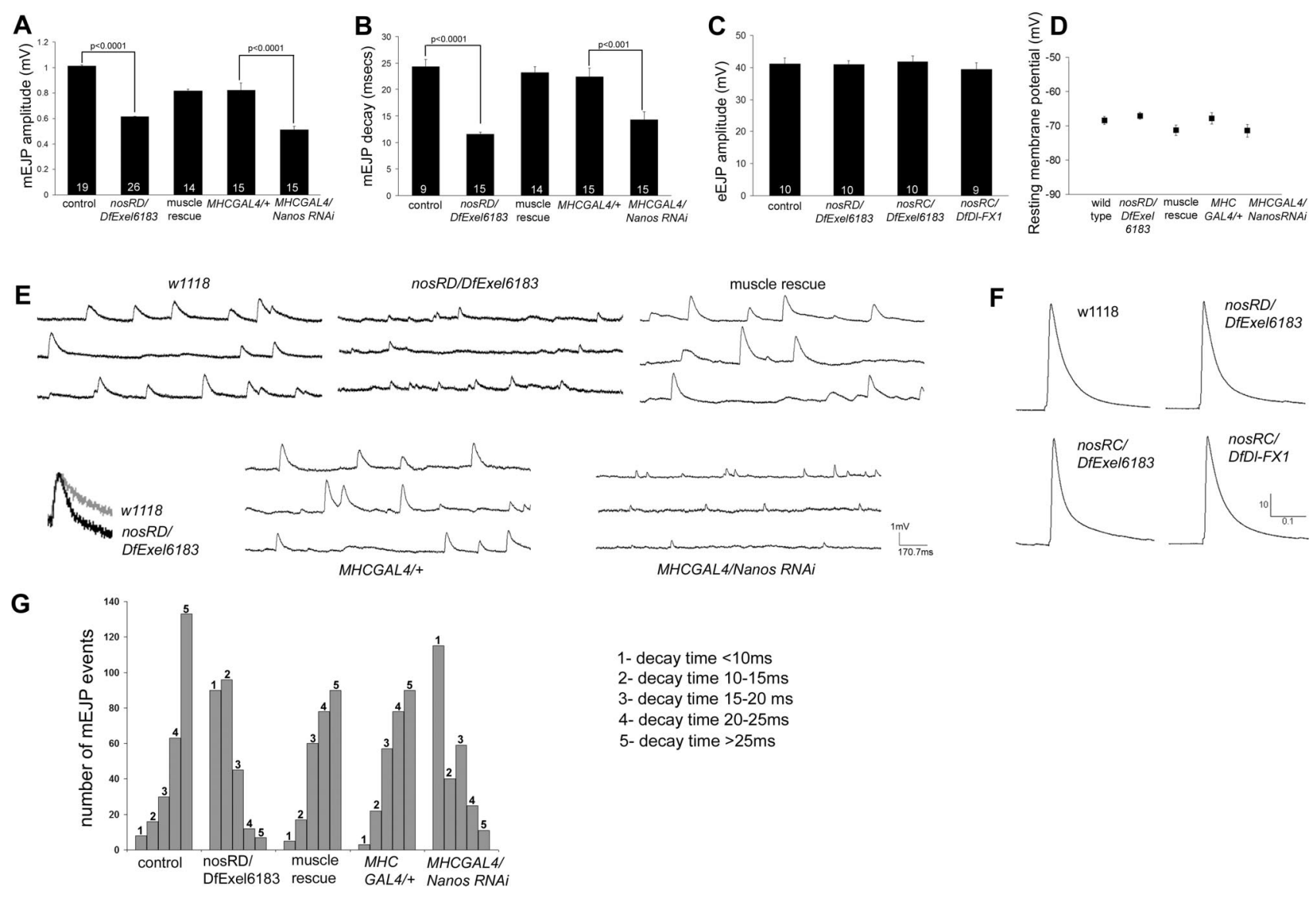

Figure 6. The amplitudes of mEJP events are decreased in nanos mutants. For electrophysiological analysis of the muscle 6/7 NMJ, recordings were done from muscle 6 in A2 and A3 segments. The number of animals that were analyzed for each genotype is indicated at the bottom of the bars. $A$, A graph of the amplitudes of mEJPs in various genotypes. Controls were two wild-type genotypes, $W^{1118}(n=10)$ and $W C S(n=9)$, and a driver crossed to $W^{1118}$ control $(n=15)$ for the RNAi animals. Amplitudes were reduced in mutants and muscle RNAi larvae. $\boldsymbol{B}$, A graph of the decay time (time constant) of mEJPs in the same genotypes. Decay was faster in mutants and muscle RNAi larvae. $\boldsymbol{C}$, A graph of eEJPs in various genotypes. There were no significant differences among genotypes. $\boldsymbol{D}, A$ graph of resting membrane potential in the five genotypes in $\boldsymbol{A}$ and $\boldsymbol{B}$. All the animals that were used for recordings had resting potentials that were more negative than $-60 \mathrm{mV}$. This indicates that all preparations were equally healthy. $\boldsymbol{E}$, Three representative traces of spontaneous events for the five genotypes in $\boldsymbol{A}$ and $\boldsymbol{B}$. To show the faster decay in the mutant compared with the control, one spontaneous event from a control $\left(w^{1118}\right.$, gray) trace and one from a nanos transheterozygote $\left[\right.$ nos $^{R D} / D f(3 R)$ Exel6183, black] trace that had the same mEJP amplitudes are shown. $\boldsymbol{F}$, Representative traces of evoked responses from the three genotypes in $\boldsymbol{C}$. Each trace is an average of 10 sequential eEJPs from one animal. $\boldsymbol{G}$, A histogram showing the distribution of decaytimes of $\mathrm{mEJP}$ events in the different genotypes. The first $50 \mathrm{mEJP}$ events from traces of five animals (a total of 250 events per genotype) were analyzed and put in bins corresponding to decay times of $<10,10-15,15-20$, $20-25$, and $>25$ ms. The mutant and muscle Nos RNAi animals have a much larger number of events with fast decay times compared with controls and muscle rescue animals.

larvae. These values were not significantly different from wild type (control: $0.8 \pm 0.09 \mathrm{mV}, n=9$; neuronal Nos RNAi: $0.79 \pm$ $0.06, n=8)$.

We measured evoked transmitter release from control and three nos transheterozygous genotypes. Evoked excitatory junctional potential (eEJP) amplitudes were similar between control and mutant animals (Fig. 6C). Traces showing evoked responses from these genotypes are shown in Figure 6F. Resting membrane potentials were approximately the same for all genotypes tested (Fig. 6D). The frequencies of spontaneous release events were not significantly different between wild type and mutants (data not shown).

The decrease in mini amplitude seen in nos mutant and muscle RNAi larvae is consistent with the expected effect of derepression of GluRIIB by loss of Nos, because GluRIIB overexpression was previously shown to result in a decrease in the amplitude of spontaneous release events. It was also demonstrated that minis from GluRIIB-dominated synapses have a faster decay time compared with GluRIIA-dominated synapses (DiAntonio et al., 1999). We thus extended our analysis to look at the decay time of spontaneous release events in the genotypes described above (Fig. $6 B$ ) (see Materials and Methods). We found that mEJPs in nos mutants had faster decay times compared with control larvae (control: $24.32 \pm 1.33 \mathrm{~ms}, n=9$; $\operatorname{nos}^{R D} / D f(3 R)$ Exel6183: $11.6 \pm$ $0.34 \mathrm{~ms}, n=15$; Student's $t$ test $p<0.0001$ ) (example traces of one spontaneous release event with the same amplitude from both genotypes are shown in Fig. $6 E$ ). The decrease in decay time was rescued by expressing Nos in the muscle in the mutant background (muscle rescue: $23.2 \pm 1.07 \mathrm{~ms}, n=14$ ). Knocking down Nos in muscles with RNAi produced a similar decrease in the decay time (control: $22.4 \pm 1.5 \mathrm{~ms}, n=15$; Nos RNAi: $14.3 \pm 1.5$ ms, $n=15$; Student's $t$ test, $p<0.001$ ). A histogram showing the distributions of decay times in the various genotypes is shown in Figure 6G. The mutant and muscle Nos RNAi animals have a much larger fraction of events with fast decay times compared with controls and muscle rescue animals.

The changes in the time constants of mEJP decay relative to controls seen in nos mutant and Nos RNAi larvae are in the range of 1.5- to 2-fold. This ratio is comparable to that reported by DiAntonio et al. (1999) for GluRIIA versus GluRIIB-dominated synapses. In summary, the electrophysiological changes we observe when postsynaptic Nos is reduced are consistent with the idea that GluRIIB synapses become the dominant type under these conditions. 

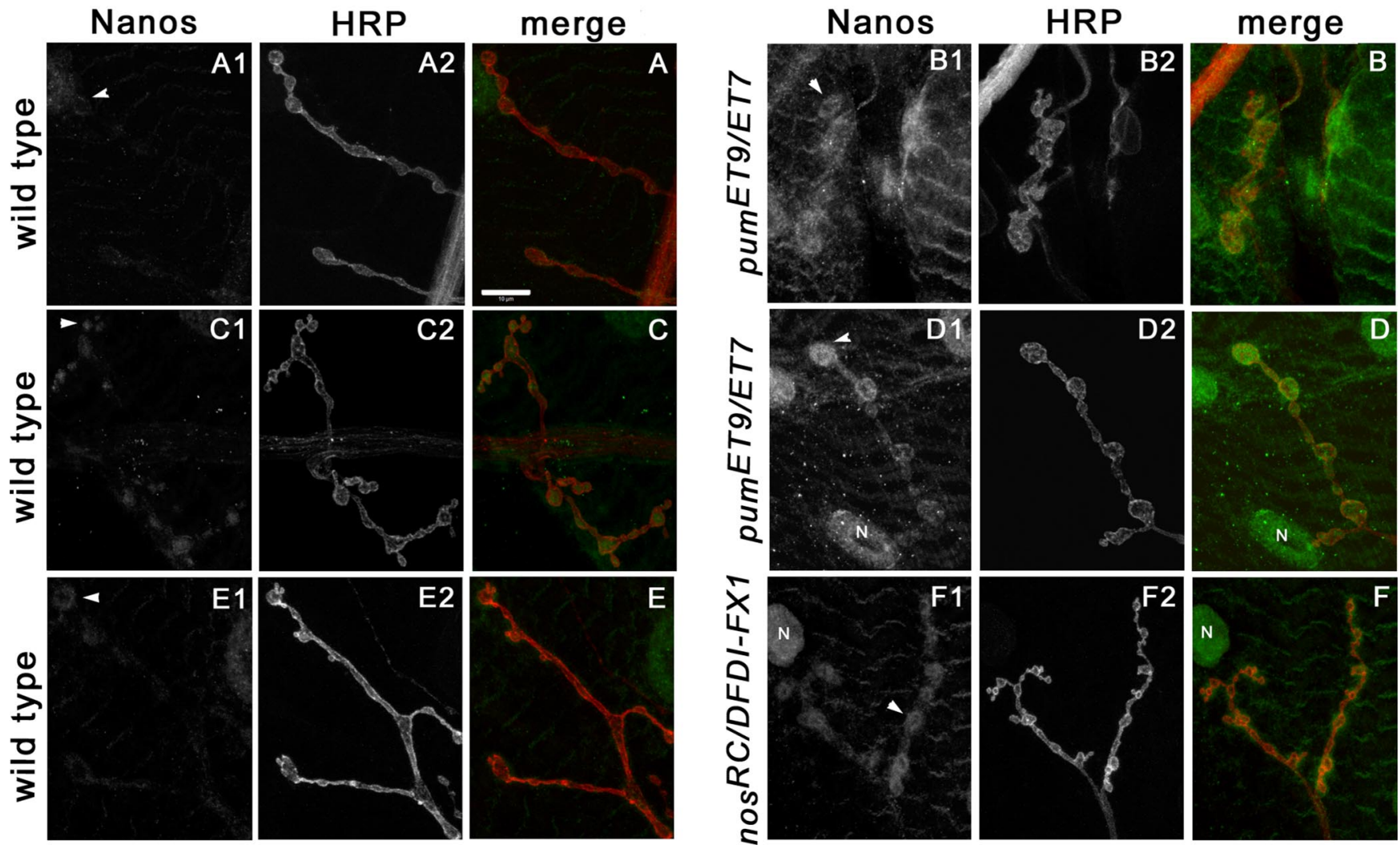

Figure 7. Nos protein expression at the NMJ is increased in pumilio and nanos mutants. Muscle 4 NMJs labeled with rat Nos antibody $(\boldsymbol{A}, \boldsymbol{B}, \boldsymbol{E}, \boldsymbol{F})$ or mouse Nos antibody $(\boldsymbol{C}, \boldsymbol{D})$ and double stained with anti-HRP. NMJs are from wild-type $\left(w^{1118} ; A, C, E\right)$, pum mutants ( pum $^{E T 7} /$ pum $\left.^{E T 9} ; \boldsymbol{B}, \boldsymbol{D}\right)$, and a nos mutant $\left[\operatorname{nos}^{R C} / D f(3 R) D I-F X 1 ; F\right] . A-D$, Both Nos antibodies reveal a large increase in staining at the postsynaptic side of the NMJ and on the muscle surface in pum mutants [compare $\boldsymbol{A} \mathbf{1}, \boldsymbol{B} \mathbf{1}$ (green in $\boldsymbol{A}$ and $\boldsymbol{B}$ for the rat Nos antibody); $\boldsymbol{C}, \mathbf{D} 2$ (green in $\boldsymbol{C}$ and $\boldsymbol{D}$ for the mouse Nos antibody)]. Segment $A 3(\boldsymbol{A}, \boldsymbol{B})$ and segment $A 2(\boldsymbol{C}, \boldsymbol{D})$ are shown. Scale bar: $\boldsymbol{A}, 10 \mu \mathrm{m} . \boldsymbol{E}, \boldsymbol{F}$, The levels of Nos-immunoreactive protein at the $\mathrm{A} 3 \mathrm{NMJ}$ and on the muscle surface are increased in this nos mutant (compare $\boldsymbol{E 1}, \boldsymbol{F 1}$; green in $\boldsymbol{E}$ and $\boldsymbol{F}$ ), as well as in two other transheterozygous nos genotypes (data not shown). Anti-HRP labeling is similar in all genotypes. The different appearance of the NMJs in $\boldsymbol{B}$ and $\boldsymbol{D}$ reflects the pum phenotype. Arrowheads indicate type $1 \mathrm{~b}$ boutons, and $\mathrm{N}$ indicates a nucleus.

\section{Postsynaptic Nos is upregulated in pumilio mutants}

GluRIIA is upregulated in pum mutants (Menon et al., 2004) (see Fig. 8), but also in muscle Nos overexpression larvae (Fig. 4). nos mRNA is downregulated in neurons when the PumRBD is overexpressed (Muraro et al., 2008). Binding of PUF-domain proteins to mRNAs does not necessarily decrease their levels, but this result does suggest that nos mRNA is affected by Pum. Also, nos mRNA was one of a large number of mRNAs that were copurified with an epitope-tagged PumRBD protein expressed in ovaries (Gerber et al., 2006). Because of these findings, we wondered whether Nos might be translationally repressed by Pum, so that Nos overexpression could contribute indirectly to the upregulation of GluRIIA observed in pum mutants as a result of the negative effect of GluRIIB on GluRIIA.

To evaluate this idea, we examined the levels of Nos in pum mutants and found that Nos staining was increased, as evaluated with both antibodies (Fig. 7, compare $A 1, B 1$ and $C 1, D 1$ ). These larvae were processed and imaged together under the same experimental conditions; they were imaged so that the Nos staining in wild type is barely detectable, to clearly display the increase in staining seen in pum mutants. Labeling with the anti-HRP marker was the same in wild type and mutant. Muscle Nos was also upregulated in larvae in which postsynaptic Pum was knocked down using a Pum RNAi line (supplemental Fig. S3, available at www.jneurosci.org as supplemental material). Nos was not upregulated in the VNC of pum mutants, suggesting that Pum does not control Nos protein levels in most larval CNS neurons (data not shown). We also examined Dlg in pum mu- tants, because it was found to be downregulated by Pum overexpression in the mushroom body (Chen et al., 2008). Dlg staining at the NMJ was not increased in pum mutants [Menon et al. (2004), their Fig. 2].

Because Nos is a corepressor for Pum in most contexts, we wondered whether Nos repression by Pum might require Nos itself. We tested this idea by examining nos mutants, which continue to make inactive Nos proteins (Verrotti and Wharton, 2000), by staining with Nos antibodies. If Nos regulates its own expression, then Nos-immunoreactive protein levels might increase in nos mutants. Confirming this prediction, Nos labeling at the NMJ in a nos mutant $\left(n o s^{R C} / D f(3 R) D l-F X 1\right)$ was increased compared with controls (Fig. 7, compare E1, F1). Three other transheterozygous nos mutant combinations were tested and produced the same result. In addition to Nos staining at the NMJ, muscle surface expression of Nos is increased in both pum and nos mutants (Fig. 7, compare green signals in merged panels). We also examined eIF-4E staining in nos mutants and found that it was unchanged (data not shown).

\section{Pum binds selectively to GluRIIA $m R N A$}

There are several possible models that could account for the increase in GluRIIA seen in pum mutants (Fig. 8A,B). First, derepression of synaptic eIF-4E (Menon et al., 2004) might cause an increase in translation of GluRIIA mRNA, which is localized to the synaptic region of the muscle fiber (Sigrist et al., 2000). Second, derepression of Nos could indirectly affect GluRIIA by downregulating GluRIIB. Third, Pum might bind directly to Glu- 
RIIA mRNA to repress its translation. To test the direct interaction model, we conducted in vitro binding experiments with the purified PumRBD. Pum binding sites have previously been identified in a number of mRNAs. For $h b$, bicoid, $C y c B$, eIF$4 E$, para, and Dlg, it has been demonstrated that Pum can regulate expression of the encoded proteins in vivo (Wharton and Struhl, 1991; Menon et al., 2004; Kadyrova et al., 2007; Chen et al., 2008; Muraro et al., 2008).

A four-nucleotide (nt) consensus motif, UGUA, is found in most Pum binding sites, and mutation of this motif disrupts Pum binding (Zamore et al., 1999). The 3'UTR of the GluRIIA mRNA contains two UGUA motifs separated by $16 \mathrm{nt}$. Therefore, to test whether Pum can directly interact with GluRIIA mRNA, we focused on this region of the 3'UTR. A $49 \mathrm{nt}$ RNA probe encompassing the two motifs was synthesized and tested for Pum binding using electrophoretic mobility shift assays (EMSA). A recombinant protein encompassing the PumRBD bound to this RNA probe, generating two complexes with different mobilities (Fig. $8 C$ ). The appearance of the upper (lower mobility) complex (Fig. 8, label 2) at higher PumRBD concentrations suggests that it resulted from binding of two PumRBD molecules to the GluRIIA probe. The interaction of the PumRBD with GluRIIA mRNA was specific, because binding could be competed away by the addition of excess unlabeled GluRIIA RNA but not by an excess of an $h b$ RNA fragment from which the NRE is deleted (Fig. $8 D, h b \mathrm{NRE}^{-}$). Interestingly, the wild-type $h b$ NRE sequence competed less effectively for binding than the GluRIIA sequence, suggesting that the GluRIIA 3'UTR may have a higher affinity for the PumRBD than does the $h b$ NRE.

To determine whether the interaction of Pum with GluRIIA sequences depends on the UGUA motifs, we generated mutant probes in which both UGUA motifs were altered to GUUA (Fig. $9 A$ ), a mutation previously shown to abolish the binding of Pum to the $h b 3^{\prime}$ UTR (Wharton et al., 1998). The GUUA mutations disrupted formation of the more slowly migrating complex (Fig. $9 B$, label 2) but had little effect on the more rapidly migrating complex (Fig. 9B, label 1). These results suggest that the double GUUA mutation prevents binding of one PumRBD molecule, since it eliminates the band with two bound PumRBDs (band 2) but preserves the band with one bound PumRBD (band 1). The other PumRBD molecule, then, binds to a distinct sequence, which we identified by making another mutation.

The Pum-related fem-3-binding factor (FBF) proteins from Caenorhabditis elegans recognize a distinct motif that also contains a core UGU triplet (Lamont et al., 2004; Bernstein et al., 2005). The GluRIIA probe sequence contains a UUGUG motif that fits the FBF binding consensus. Mutation of this sequence (M2) in the GluRIIA probe also prevented formation of the complex with two Pum molecules (band 2), but not the complex with one molecule (band 1) (Fig. 9B). Thus, a GluRIIA probe with a mutation in either the UGUA or M2 sites binds to one PumRBD molecule, whereas a wild-type probe binds to two PumRBDs. Together, these results are consistent with binding of Pum to two different motifs within the GluRIIA 3'UTR: a canonical UGUA motif and an FBF site-like recognition motif.

\section{Pum binds selectively to the nanos $3^{\prime}$ UTR}

In pum mutants, the levels of eIF-4E, GluRIIA, and Nos are all increased, and we have shown that Pum binds selectively to the eIF-4E (Menon et al., 2004) and GluRIIA 3'UTRs (Fig. 8). We thus wondered whether the nos 3'UTR might also be a direct Pum target. Sequences that mediate localization and translational control of nos mRNA in the oocyte, embryo, and PNS reside within the nos 3'UTR (Gavis and Lehmann, 1992, 1994; Forrest et al., 2004; Brechbiel and Gavis, 2008). A single UGUA motif is present in the nos 3'UTR, in a region with no previously identified regulatory function. We surveyed fragments spanning the nos $3^{\prime} \mathrm{UTR}$ for Pum binding using a UV-crosslinking assay. Only one fragment (nos 3'UTR nucleotides 403-553) was bound by the PumRBD (Fig. 9C). Notably, PumRBD did not crosslink to a fragment containing the UGUA motif (data not shown), indicating that Pum interacts with the nos 3'UTR through one or more alternative binding sites.

ClustalW alignment of sequences from the GluRIIA and nos 3'UTR fragments bound by Pum identified a $31 \mathrm{nt}$ region with $58 \%$ sequence identity (common nucleotides indicated in red in 
GIURIIA GUGUCCACAUUGUAUAUUGUGAGCUAUAUUUGUAGAAUUAUUUU

GluRIIA GUUA GUGUCCACAUGUUAUAUUGUGAGCUAUAUUGUUAGAAUUAUUUU

GIURIIA M2 GUGUCCACAUUGUAUCUGAGACGCUAUAUUUGUAGAAUUAUUUU

nos CACUUUGAAUUUCGAACUGUCAAUCGUAUCAUUAGAAUUUAAUC
nos M1 CACUUUGAGCCUCGAACUGUCAAUCGUAUCAUUAGAAUUUAAUC
nos M2 CACUUUGAAUUUCGACUGAGACAUCGUAUCAUUAGAAUUUAAUC
nos M3 CACUUUGAAUUUCGAACUGUCAAUCGUAUCAUGCCCGGGUAUC

elF-4E CCGCUUUUUGUAGUCACUGUCAAUAAUGGUAUUA

nos CACUUUGAAUUUCGAACUGUCAAUCGUAUCAUUAGAAUUUAAUC

GluRIIA GUGUCCACAUUGUAUAUUGUGAGCUAUAUUUGUAGAAUUAUUUU
C

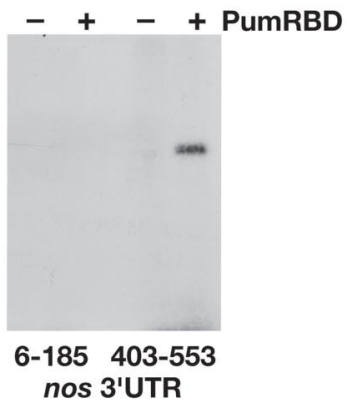

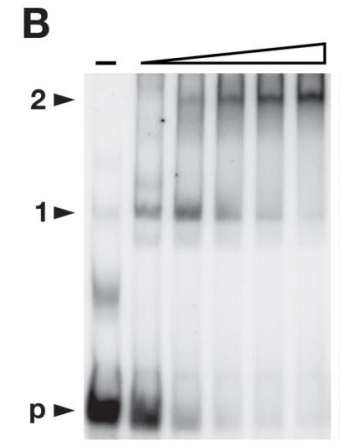

GIURIIA WT

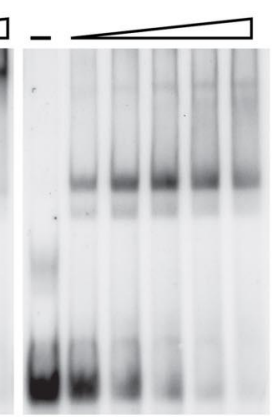

GIURIIA GUUA

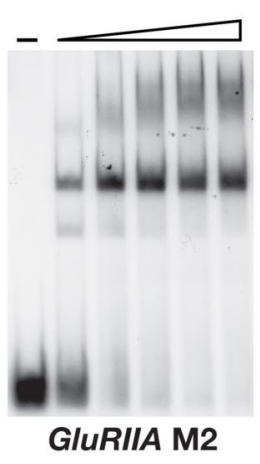

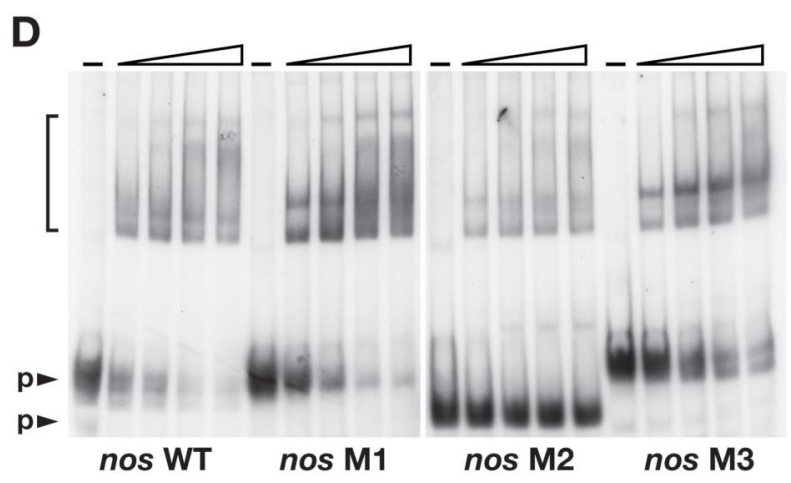

Figure 9. Pum recognizes conserved noncanonical binding sites in the nanos and GluRIIA 3'UTRs. A, The sequences of the GluRIIA and nos $405-453$ fragments bound by Pum, with nucleotides conserved between the two indicated in red. The sequences of the GluRIIA GUUA and $M 2$ mutants and the nos $M 1, M 2$, and $M 3$ mutants are shown below the respective wild-type sequences, with altered nucleotides in green. The lower alignment shows similarity among the Pum-binding regions of the elF-4E, nos, and GIURIIA $3^{\prime}$ UTRs. Note that there is a $9 \mathrm{nt}$ sequence spanning the M2 motif that is identical between elF-4E and nos. B, Gel mobility shift assays of PumRBD binding to ${ }^{32} \mathrm{P}$-labeled GluRIIA wild-type, GUUA, or M2 mutant RNA probes. Binding reactions contained the same amount of RNA probe and either $0(-)$ or an increasing concentration of PumRBD protein (ramp above lanes). $C$, UV-crosslinking of the PumRBD to ${ }^{32}$ P-labeled nos RNA probes. Pum binds to a fragment encompassing nos 3'UTR nucleotides 405-453, but not to a fragment containing nucleotides $6-185$. - , Reaction without PumRBD; +, reaction containing PumRBD. D, Gel mobility shift assays of PumRBD binding to ${ }^{32} \mathrm{P}$-labeled nos wild-type, M1, M2, and M3 mutant RNA probes. Binding reactions contained the same amount of RNA probe and either 0 ( - ) or an increasing concentration of PumRBD protein. The Pum-nos RNA complex migrates as a smear of several bands (bracket); the overall intensity of this smear is decreased in the M2 lanes but not for the other mutants. Note also that the unbound M2 mutant probe is not depleted even at the highest concentration of PumRBD, whereas the other probes are. The reason for the differing mobility of the M2 probe is not known but presumably reflects an inherent sequence and/or structural difference. WT, Wild type.

Fig. 9A). The PumRBD bound to a $50 \mathrm{nt}$ probe encompassing the nos sequence, as assayed by EMSA, suggesting that the conserved nucleotides represent a Pum binding site. To test this hypothesis, we generated three sets of mutations that alter conserved sequences within the nos 3'UTR fragment (Fig. 9A) and assayed the effect of these mutations on Pum binding. The nos M1 and M3 mutations had no significant effect on Pum binding relative to the wild-type sequence (Fig. 9D). However, the ability of Pum to interact with the nos sequence was diminished by the M2 mutation. This decrease in binding is detected as a diminution in the intensity of the complex bands, coupled to a failure of complex formation to deplete the unbound probe [Fig. 9D, compare intensity of the unbound bands (arrowheads) in the right two nos-M2 lanes with the corresponding lanes for wild-type, M1, and M3 mutants). The M2 mutation disrupts a UGU triplet that corresponds to the FBF-like binding site in GluRIIA. These results indicate that Pum recognizes the nos $3^{\prime} \mathrm{UTR}$, as well as the GluRIIA 3'UTR, using a motif that differs from the UGUA consensus. Remarkably, a $9 \mathrm{nt}$ sequence spanning M2 is identical to a sequence within the $51 \mathrm{nt}$ minimal Pum-binding fragment of the eIF-4E 3'UTR (Menon et al., 2004). This suggests that M2-like sequences may represent a new type of synaptic Pum binding site.

\section{Discussion}

Nos and Pum are translational repressors that are best known for their roles in early development. Maternal Nos and Pum form a complex that binds to $h b$ mRNA, which is normally translated only in the anterior part of the embryo. When Nos or Pum are absent, $\mathrm{Hb}$ protein is made throughout the embryo, and this produces segmental deletions.

Nos and Pum are required for nervous system development, and results published previously are consistent with the idea that they also work together to repress translation in these contexts (Ye et al., 2004; Muraro et al., 2008). We have focused on the neuromuscular system, finding phenotypes and targets for Pum at the larval NMJ (Menon et al., 2004). In this study, we examine Nos. Nos, like Pum, is expressed in neuronal cell bodies, at the NMJ, and in the muscles (Fig. 1). However, Nos and Pum have divergent functions in NMJ development. In nos mutant and neuronal Nos RNAi larvae, the number of boutons at the NMJ is increased, but their size is unaltered (Figs. 2, 3). In contrast, loss of Pum from neurons causes a bouton fusion phenotype, so that $1 \mathrm{~b}$ boutons are fewer in number and much larger (Menon et al., 2004).

Overexpression of Nos in neurons generates a phenotype in 
which the number of AZs in type 1s boutons is reduced (Fig. 3). Pum neuronal overexpression, however, reduces bouton size and increases bouton number, so that the NMJ becomes a cluster of tiny boutons (Menon et al., 2004). The Pum overexpression phenotype resembles that seen for loss-of-function mutants affecting proteins in endocytic pathways, such as endophilin, synaptojanin, dynamin, and Synaptotagmin (Dickman et al., 2006), suggesting that one or more of these might be neuronal Pum targets.

In pum mutants, the GluRIIA subunit of the muscle glutamate receptor is dramatically upregulated (Menon et al., 2004) (Fig. 8). In muscle Nos RNAi larvae, however, GluRIIA is downregulated (Fig. 4), and the levels of the alternative subunit GluRIIB at the NMJ are increased (Fig. 5). In larvae overexpressing Nos in muscles, GluRIIA levels are increased (Fig. 4). The data suggest that postsynaptic Nos represses GluRIIB and induces GluRIIA and that its effects on GluRIIB levels are, at least partially, independent of GluRIIA (Fig. 5).

The electrophysiological effects of loss of postsynaptic Nos are consistent with this model, because mEJP amplitudes are decreased, and the spontaneous events have fast decay kinetics (Fig. 6). These are known properties of GluRIIB-containing synapses (DiAntonio et al., 1999).

PumRBD overexpression causes downregulation of nos mRNA in neurons (Muraro et al., 2008), and nos was identified as a PumRBD-bound mRNA in a screen for ovary mRNAs bound to a tagged Pum protein in vivo (Gerber et al., 2006). Based on these findings, we examined the relationships between Nos and Pum in our system. We found that Nos is a target for Pum repression in muscles (Fig. 7) and that the PumRBD binds selectively to nos mRNA in vitro (Fig. 9).

\section{Translational repression by Pum and Nos}

In the early embryo, which is a syncytium, Pum is distributed throughout the cytoplasm, whereas Nos is selectively translated from mRNA localized to the posterior pole. Nos protein diffuses anteriorly into the embryo, forming a transient gradient. Pum binds to NREs in the 3'UTR of $h b$ mRNA, but Nos does not exhibit sequencespecific RNA binding in the absence of Pum (for review, see Kuersten and Goodwin, 2003). Pum/Nos bound to the $h b$ NRE recruits Brat, and the Pum/Nos/Brat ternary complex represses translation of $h b$ mRNA (Sonoda and Wharton, 2001).

Pum is a founding member of the PUF family of translational repressors (for review, see Wickens et al., 2002). PUF proteins bind to Pop2p and its orthologs (Goldstrohm et al., 2006, 2007; Kadyrova et al., 2007). Pop2p is a deadenylase and is a component of a complex that also includes decapping proteins. The complex might thus remove all or part of the polyA tail (thereby destabilizing the mRNA) and also remove the cap (thereby blocking translational initiation) from any mRNA to which it is recruited by Pum binding. However, this is not sufficient for translational repression of $h b \mathrm{mRNA}$, since Nos and Brat are also required.

Pum and Nos also repress translation of $C y c B$ mRNA in germ cells, which do not express Brat. Nos can recruit another subunit of a deadenylase/decapping complex, NOT4, and artificial tethering of Nos to $C y c B$ mRNA in the absence of Pum is sufficient for repression in germ cells (Kadyrova et al., 2007). These data show that Nos can repress translation without Pum if it is brought to an mRNA target by another sequence-specific binding protein.

\section{Direct regulation of postsynaptic targets by Pum}

eIF-4E is dramatically upregulated at the NMJ in pum mutants. The $3^{\prime}$ UTR of eIF-4E mRNA contains a high-affinity Pum bind- ing site, suggesting that it is a direct repression target (Menon et al., 2004). eIF-4E is not upregulated in nos mutants, indicating that Pum affects eIF-4E translation by recruiting other corepressors to the mRNA. Similarly, GluRIIA is elevated in pum mutants but is not increased when Nos is knocked down in muscles (Figs. $4,8)$. We had originally speculated that GluRIIA upregulation in pum mutants might be an indirect effect caused by elevation of postsynaptic eIF-4E. Here, however, we show that Pum also binds directly to the $3^{\prime} \mathrm{UTR}$ of GluRIIA mRNA (Fig. 8). The GluRIIA minimal binding region has two Pum sites. One of these contains the UGUA motif, which is critical for Pum binding to the $h b$ NRE. The second site, M2, is eliminated by mutations affecting UGUG, which is found within sites for a different PUF domain protein, C. elegans FBF (Fig. 9) (Zamore et al., 1999; Lamont et al., 2004; Bernstein et al., 2005).

Pum also binds directly to the $3^{\prime} \mathrm{UTR}$ of nos mRNA. The nos minimal binding region does not contain a UGUA, and Pum binding is reduced by mutation of an M2-like sequence. The M2 sequences in GluRIIA and nos are similar to a sequence in the eIF-4E minimal binding region, suggesting that these $3^{\prime}$ UTR sites may represent a new type of postsynaptic Pum-binding element (Fig. 9). However, the nos 3'UTR, unlike the eIF-4E and GluRIIA 3'UTRs, is likely to be a target for the Pum/Nos repression complex, because Nos protein is upregulated in nos mutants (Fig. 7).

\section{A model for regulation of synaptic translation and physiology by Pum and Nos}

The NMJ glutamate receptor is composed of three invariant subunits (GluRIIC, GluRIID, and GluRIIE), together with either GluRIIA or GluRIIB (Marrus et al., 2004; Qin et al., 2005). An AZ is associated with clusters of both GluRIIA and GluRIIB subunitcontaining receptors (Rasse et al., 2005; Schmid et al., 2008). GluRIIA receptors admit more current in response to transmitter release than GluRIIB receptors (DiAntonio et al., 1999; Sigrist et al., 2003; Pawlu et al., 2004). GluRIIA and GluRIIB compete with each other for occupancy within synaptic receptor complexes, so that overexpression of the GluRIIB subunit causes a decrease in the number of GluRIIA-containing receptors at the synapse, and vice versa (Petersen et al., 1997; DiAntonio et al., 1999; Sigrist et al., 2002) [but see Schmid et al. (2008) for an alternative view].

When larval motility is induced by a shift from liquid to solid medium, synaptic strength increases by $40 \%$ over a $3 \mathrm{~h}$ period. GluRIIA and postsynaptic eIF-4E are both upregulated during this time period (Sigrist et al., 2000, 2003). By 24 h later, new boutons are added to the NMJ. It has been proposed that elevation of postsynaptic translation in response to the need for increased movement is used to facilitate rapid synaptic growth ( $\mathrm{Si}$ grist et al., 2002). Pum appears to be an upstream component of this process, because postsynaptic eIF-4E and GluRIIA are derepressed in pum mutants even without induction of motility (Menon et al., 2004). This suggests that the induction of synaptic translation and the shift from GluRIIB to GluRIIA receptors that occurs in normal larvae in response to increased movement could be triggered by partial inactivation or degradation of Pum.

The regulation of Nos expression by postsynaptic Pum should amplify the effects on synaptic physiology of a reduction in Pum activity. This is because reducing Pum both directly derepresses GluRIIA expression (Fig. 8) and also increases Nos, which represses GluRIIB (Fig. 5). In this manner, a relatively small decrease in Pum levels could be amplified into a large change in the ratio of GluRIIA to GluRIIB in synaptic receptor complexes (Fig. 10). This model leaves open the question of how Nos represses GluRIIB without Pum. Nos might be recruited to postsynaptic 


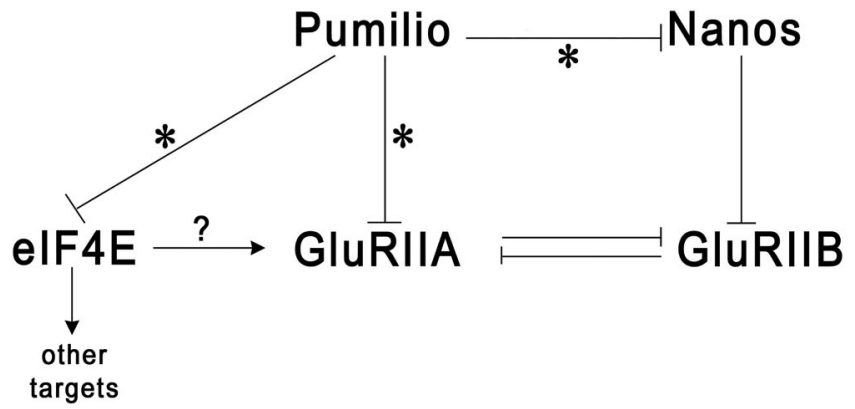

Figure 10. A diagram of the regulatory interactions among Pum, Nos, GluRIIA, GluRIIB, and elF-4E. Pum represses elF-4E, Nos, and GluRIIA and directly binds to all three target mRNAs (*). elF-4E is often limiting for translation, so its elevation in pum mutants may help stimulate translation of GluRIIA and other postsynaptic mRNAs. Others have shown that GluRIIA and GluRIIB have mutually repressive interactions (see Discussion for references). This diagram predicts that the regulatory interplay among these factors should act as an amplifier, converting a small decrease in Pum activity (possibly in response to changes in environmental conditions) into a large shift in the GluRIIA/GluRIIB ratio.

mRNA targets by interaction with another sequence-specific binding protein(s). Alternatively (or in addition), Nos might indirectly control GluRIIB by regulating signaling pathways that affect receptor composition or localization.

\section{References}

Bergsten SE, Huang T, Chatterjee S, Gavis ER (2001) Recognition and longrange interactions of a minimal nanos RNA localization signal element. Development 128:427-435.

Bernstein D, Hook B, Hajarnavis A, Opperman L, Wickens M (2005) Binding specificity and mRNA targets of a C. elegans PUF protein, FBF-1. RNA 11:447-458.

Brand AH, Perrimon N (1993) Targeted gene expression as a means of altering cell fates and generating dominant phenotypes. Development 118:401-415.

Brechbiel JL, Gavis ER (2008) Spatial regulation of nanos is required for its function in dendrite morphogenesis. Curr Biol 18:745-750.

Chen G, Li W, Zhang QS, Regulski M, Sinha N, Barditch J, Tully T, Krainer AR, Zhang MQ, Dubnau J (2008) Identification of synaptic targets of Drosophila pumilio. PLoS Comput Biol 4:e1000026.

Cho PF, Gamberi C, Cho-Park YA, Cho-Park IB, Lasko P, Sonenberg N (2006) Cap-dependent translational inhibition establishes two opposing morphogen gradients in Drosophila embryos. Curr Biol 16:2035-2041.

Curtis D, Treiber DK, Tao F, Zamore PD, Williamson JR, Lehmann R (1997) A CCHC metal-binding domain in Nanos is essential for translational regulation. EMBO J 16:834-843.

DiAntonio A, Petersen SA, Heckmann M, Goodman CS (1999) Glutamate receptor expression regulates quantal size and quantal content at the Drosophila neuromuscular junction. J Neurosci 19:3023-3032.

Dickman DK, Lu Z, Meinertzhagen IA, Schwarz TL (2006) Altered synaptic development and active zone spacing in endocytosis mutants. Curr Biol 16:591-598.

Dietzl G, Chen D, Schnorrer F, Su KC, Barinova Y, Fellner M, Gasser B, Kinsey K, Oppel S, Scheiblauer S, Couto A, Marra V, Keleman K, Dickson BJ (2007) A genome-wide transgenic RNAi library for conditional gene inactivation in Drosophilia. Nature 448:151-156.

Forbes A, Lehmann R (1998) Nanos and Pumilio have critical roles in the development and function of Drosophila germline stem cells. Development 125:679-690.

Forrest KM, Clark IE, Jain RA, Gavis ER (2004) Temporal complexity within a translational control element in the nanos mRNA. Development 131:5849-5857.

Gavis ER, Lehmann R (1992) Localization of nanos RNA controls embryonic polarity. Cell 71:301-313.

Gavis ER, Lehmann R (1994) Translational regulation of nanos by RNA localization. Nature 369:315-318.

Gavis ER, Curtis D, Lehmann R (1996a) Identification of cis-acting sequences that control nanos RNA localization. Dev Biol 176:36-50.

Gavis ER, Lunsford L, Bergsten SE, Lehmann R (1996b) A conserved 90 nucleotide element mediates translational repression of nanos RNA. Development 122:2791-2800.

Gerber AP, Luschnig S, Krasnow MA, Brown PO, Herschlag D (2006) Genome-wide identification of mRNAs associated with the translational regulator PUMILIO in Drosophila melanogaster. Proc Natl Acad Sci U S A 103:4487-4492.

Goldstrohm AC, Hook BA, Seay DJ, Wickens M (2006) PUF proteins bind Pop2p to regulate messenger RNAs. Nat Struct Mol Biol 13:533-539.

Goldstrohm AC, Seay DJ, Hook BA, Wickens M (2007) PUF proteinmediated deadenylation is catalyzed by Ccr4p. J Biol Chem 282:109-114

Kadyrova LY, Habara Y, Lee TH, Wharton RP (2007) Translational control of maternal Cyclin B mRNA by Nanos in the Drosophila germline. Development 134:1519-1527.

Kalifa Y, Huang T, Rosen LN, Chatterjee S, Gavis ER (2006) Glorund, an $\mathrm{hnRNP} \mathrm{F} / \mathrm{H}$ homolog, is an ovarian repressor of nanos translation. Dev Cell 10:291-301.

Kittel RJ, Wichmann C, Rasse TM, Fouquet W, Schmidt M, Schmid A, Wagh DA, Pawlu C, Kellner RR, Willig KI, Hell SW, Buchner E, Heckmann M, Sigrist SJ (2006) Bruchpilot promotes active zone assembly, Ca2+ channel clustering, and vesicle release. Science 312:1051-1054.

Kuersten S, Goodwin EB (2003) The power of the 3' UTR: translational control and development. Nat Rev Genet 4:626-637.

Lamont LB, Crittenden SL, Bernstein D, Wickens M, Kimble J (2004) FBF-1 and FBF-2 regulate the size of the mitotic region in the C. elegans germline. Dev Cell 7:697-707.

Lee YS, Carthew RW (2003) Making a better RNAi vector for Drosophila: use of intron spacers. Methods 30:322-329.

Marrus SB, Portman SL, Allen MJ, Moffat KG, DiAntonio A (2004) Differential localization of glutamate receptor subunits at the Drosophila neuromuscular junction. J Neurosci 24:1406-1415.

Mee CJ, Pym EC, Moffat KG, Baines RA (2004) Regulation of neuronal excitability through pumilio-dependent control of a sodium channel gene. J Neurosci 24:8695-8703.

Menon KP, Sanyal S, Habara Y, Sanchez R, Wharton RP, Ramaswami M, Zinn K (2004) The translational repressor Pumilio regulates presynaptic morphology and controls postsynaptic accumulation of translation factor eIF-4E. Neuron 44:663-676.

Muraro NI, Weston AJ, Gerber AP, Luschnig S, Moffat KG, Baines RA (2008) Pumilio binds para mRNA and requires Nanos and Brat to regulate sodium current in Drosophila motoneurons. J Neurosci 28:2099-2109.

Osterwalder T, Yoon KS, White BH, Keshishian H (2001) A conditional tissue-specific transgene expression system using inducible GAL4. Proc Natl Acad Sci U S A 98:12596-12601.

Pawlu C, DiAntonio A, Heckmann M (2004) Postfusional control of quantal current shape. Neuron 42:607-618.

Petersen SA, Fetter RD, Noordermeer JN, Goodman CS, DiAntonio A (1997) Genetic analysis of glutamate receptors in Drosophila reveals a retrograde signal regulating presynaptic transmitter release. Neuron 19:1237-1248.

Qin G, Schwarz T, Kittel RJ, Schmid A, Rasse TM, Kappei D, Ponimaskin E, Heckmann M, Sigrist SJ (2005) Four different subunits are essential for expressing the synaptic glutamate receptor at neuromuscular junctions of Drosophila. J Neurosci 25:3209-3218.

Rasse TM, Fouquet W, Schmid A, Kittel RJ, Mertel S, Sigrist CB, Schmidt M, Guzman A, Merino C, Qin G, Quentin C, Madeo FF, Heckmann M, Sigrist SJ (2005) Glutamate receptor dynamics organizing synapse formation in vivo. Nat Neurosci 8:898-905.

Schmid A, Sigrist SJ (2008) Analysis of neuromuscular junctions: histology and in vivo imaging. Methods Mol Biol 420:239-251.

Schmid A, Hallermann S, Kittel RJ, Khorramshahi O, Frolich AM, Quentin C, Rasse TM, Mertel S, Heckmann M, Sigrist SJ (2008) Activity-dependent site-specific changes of glutamate receptor composition in vivo. Nat Neurosci 11:659-666.

Sigrist SJ, Thiel PR, Reiff DF, Lachance PE, Lasko P, Schuster CM (2000) Postsynaptic translation affects the efficacy and morphology of neuromuscular junctions. Nature 405:1062-1065.

Sigrist SJ, Thiel PR, Reiff DF, Schuster CM (2002) The postsynaptic glutamate receptor subunit DGluR-IIA mediates long-term plasticity in Drosophila. J Neurosci 22:7362-7372.

Sigrist SJ, Reiff DF, Thiel PR, Steinert JR, Schuster CM (2003) Experiencedependent strengthening of Drosophila neuromuscular junctions. J Neurosci 23:6546-6556. 
Sonoda J, Wharton RP (2001) Drosophila Brain Tumor is a translational repressor. Genes Dev 15:762-773.

Verrotti AC, Wharton RP (2000) Nanos interacts with cup in the female germline of Drosophila. Development 127:5225-5232.

Wagh DA, Rasse TM, Asan E, Hofbauer A, Schwenkert I, Durrbeck H, Buchner S, Dabauvalle MC, Schmidt M, Qin G, Wichmann C, Kittel R, Sigrist SJ, Buchner E (2006) Bruchpilot, a protein with homology to ELKS/ CAST, is required for structural integrity and function of synaptic active zones in Drosophila. Neuron 49:833-844.

Wharton RP, Struhl G (1991) RNA regulatory elements mediate control of Drosophila body pattern by the posterior morphogen nanos. Cell 67:955-967.

Wharton RP, Sonoda J, Lee T, Patterson M, Murata Y (1998) The Pumilio
RNA-binding domain is also a translational regulator. Mol Cell $1: 863-872$.

Wickens M, Bernstein DS, Kimble J, Parker R (2002) A PUF family portrait: 3'UTR regulation as a way of life. Trends Genet 18:150-157.

Ye B, Petritsch C, Clark IE, Gavis ER, Jan LY, Jan YN (2004) Nanos and Pumilio are essential for dendrite morphogenesis in Drosophila peripheral neurons. Curr Biol 14:314-321.

Zamore PD, Williamson JR, Lehmann R (1997) The Pumilio protein binds RNA through a conserved domain that defines a new class of RNAbinding proteins. RNA 3:1421-1433.

Zamore PD, Bartel DP, Lehmann R, Williamson JR (1999) The PUMILIORNA interaction: a single RNA-binding domain monomer recognizes a bipartite target sequence. Biochemistry 38:596-604. 